\title{
Transient Evolution of Langmuir Turbulence in Ocean Boundary Layers Driven by Hurricane Winds and Waves
}

\author{
Peter P. Sullivan \\ National Center for Atmospheric Research, Boulder, Colorado \\ LEONEL ROMERO* \\ Scripps Institution of Oceanography, La Jolla, California \\ JAMES C. MCWiLLIAMS \\ Department of Atmospheric and Oceanic Science, University of California, Los Angeles, Los Angeles, California \\ W. Kendall MelviLle \\ Scripps Institution of Oceanography, La Jolla, California
}

(Manuscript received 3 February 2012, in final form 5 June 2012)

\begin{abstract}
A large-eddy simulation (LES) model, which adopts wave-averaged equations with vortex force, is used to investigate Langmuir turbulence and ocean boundary layer (OBL) dynamics in high-wind hurricane conditions. The temporally evolving spatially asymmetric wind and wave Stokes drift velocity imposed in the LES are generated by a spectral wave prediction model adapted to Hurricane Frances traveling at a speed of $5.5 \mathrm{~m} \mathrm{~s}^{-1}$. The potency of Langmuir turbulence depends on the turbulent Langmuir number, the wind-Stokes drift alignment, and the depth scale of the Stokes profile $D_{s}$ relative to the OBL depth $h$. At the time of maximum winds, large-scale vigorous coherent cells develop on the right-hand side of the storm under the inertially rotating winds; the Stokes drift velocity is well tuned to the surface winds. Much weaker cells develop on the left-hand side of the storm, partly because of reduced Stokes production. With misaligned winds and waves the vertical momentum fluxes can be counter to the gradient of Stokes drift, and the cell orientation tracks the direction of the mean Lagrangian shear. The entrainment flux is increased by $20 \%$ and the sea surface temperature is $0.25 \mathrm{~K}$ cooler on the right-hand side of the storm in the presence of Langmuir turbulence. Wave effects impact entrainment when the ratio $D_{s} /|h|>0.75$. Because of wind-wave asymmetry Langmuir cells add quantitatively to the left-right asymmetry already understood for hurricanes due to resonance. And the transient evolution of the OBL cannot be understood simply in terms of equilibrium snapshots.
\end{abstract}

\section{Introduction}

The interaction between winds, waves, and currents ultimately determines the fate of turbulent mixing in the upper ocean boundary layer (OBL). There is a growing

\footnotetext{
* Current affiliation: Marine Science Institute, University of California, Santa Barbara, Santa Barbara, California.

Corresponding author address: Peter P. Sullivan, MMM Division, NCAR, Boulder, CO 80307-3000.

E-mail:pps@ucar.edu
}

body of large-eddy simulation (LES) modeling studies supported by observational evidence that finds surface waves, and in particular phase-averaged wave-current interactions and stochastic wave breaking, play important roles in the dynamics of the upper ocean boundary layer (Sullivan and McWilliams 2010). The most compelling theoretical model of wave-current interactions is embodied in wave-averaged equations and their mathematical representation by a vortex force (Craik and Leibovich 1976; Leibovich 1983; McWilliams et al. 1997; McWilliams and Restrepo 1999; McWilliams et al. 2004). The latter is found to generate coherent Langmuir cells on a background of OBL turbulence that enhance turbulence 
kinetic energy and promote entrainment at the thermocline (Skyllingstad and Denbo 1995; McWilliams et al. 1997). Wave breaking can modulate the formation of these coherent structures, and breaking energetics increase dissipation rates in the upper OBL by several orders of magnitude compared to classic law-of-the-wall scaling (Terray et al. 1996; Sullivan et al. 2007; McWilliams et al. 2012). The regime where surface waves play an important role in the dynamics of the OBL is now referred to as Langmuir turbulence (McWilliams et al. 1997). Langmuir turbulence is created during the passage of storms, cold fronts, and tropical cyclones. These intermittent high-impact weather events, which help define the overall ocean structure and global sea surface temperature (SST), are subgrid-scale to the current generation of coupled-climate models, and thus improved parameterizations of OBLs with wave effects are generally needed (Sullivan and McWilliams 2010; Belcher et al. 2012).

Observations of wavy upper-ocean dynamics are conducted in the presence of broadband wind wave complexity, for example, wind wave disequilibrium, persistent swell, high winds with inertially rotating wind stress, and sharp fronts and submesoscale motions with breaking waves are common (Smith 1998; D'Asaro 2001; Melville et al. 2005; Capet et al. 2008; Sanford et al. 2011). LES studies of the OBL that include vortex force are, however, almost exclusively conducted for idealized steady flow regimes. Essentially, lengthy LES time integrations are carried out assuming a false independence of winds and waves; that is, there is no wind wave evolution over the long time periods used to gather turbulence statistics. This is an acceptable approximation for near wind-wave equilibrium conditions but is less certain in nonstationary wave states. Thus, a next step in complexity is exploring the dynamics of Langmuir turbulence in an evolving wind wave regime. Recent LES investigations consider wavy OBLs with a modest degree of nonequilibrium wind wave forcing, for example, idealized wind-wave misalignment (Van Roekle et al. 2012), nonstationary mixing in a shallow ocean (Kukulka et al. 2010), evolution of Langmuir turbulence over a range of wind speeds and wave age (Harcourt and D'Asaro 2008), and wind-driven mixing below the thermocline (Grant and Belcher 2011).

The present study expands our previous LES modeling investigations of Langmuir turbulence to the high wind (hurricane) driven ocean boundary layer. This regime features rapidly turning wind stress and temporally evolving wave fields that are a complex interacting mix of wind waves and swell with multiple spectral peaks propagating in directions misaligned with the surface winds (Wright et al. 2001; Fan et al. 2009). The hurricaneforced OBL then allows an assessment of Langmuir turbulence in a highly nonequilibrium wind wave regime. The classic study of Price (1981) and more recently Sanford et al. (2011) emphasize the importance of inertial resonance for ocean mixing, that is, wind stress rotating in the same direction as the underlying currents. These studies, however, do not consider OBL dynamics in the presence of the companion significant surface wave fields that high winds generate.

The goal of the present work is to examine the response of the wavy OBL to realistic time varying wind and wave forcing induced by an idealized moving hurricane. The previous LES investigation most closely related to the present work is that of Skyllingstad et al. (2000) who examined OBLs driven by simplified monochromatic wave fields and perfectly resonant wind stress, that is, a time-varying surface stress with period $T=2 \pi / f$ in which $f$ is the Coriolis parameter. A preliminary LES of a hurricane OBL with a simplified time-varying wave field is described by Sullivan and McWilliams (2010). There are numerous studies of high-wind OBLs carried out using 1D single-column parameterizations (e.g., Price et al. 1986; Large et al. 1994; Crawford and Large 1996; Zedler et al. 2002). These investigations emphasize the importance of inertially rotating wind stress for mixing at the thermocline but do not explicitly include surface wave effects; wave effects may be indirectly included in these models through empirically tuned constants and mixing rules. The computational elements of the present problem are a spectral wave prediction model WaveWatch III (Tolman 2002; Romero and Melville 2010b) and an LES model of the OBL with wave effects (Sullivan et al. 2007). The manuscript is arranged as follows: section 2 describes the large-scale hurricane wind and wave fields; sections 3 and 4 provide an overview of the problem posing and discuss the external wind and wave forcings applied to the LES model; sections 5 and 6 are devoted to a discussion of the results; and section 7 is a summary of the findings.

\section{Hurricane wind and wave fields}

Present computer power is insufficient to simultaneously resolve all dynamical scales in a hurricane-driven ocean basin where the largest scales of motion are hundreds of kilometers in horizontal directions while the smallest scales associated with Langmuir turbulence are about one meter. To make our small-scale turbulence computations tractable, we utilize a simplified one-way coupling strategy where the wave fields are first computed over a large ocean basin using surface winds that are an idealization of a translating hurricane vortex. In the present study, the wind fields come from hindcast products and are an idealization of Hurricane Frances. 
The winds are first used to drive the wave model, and then the Stokes drift, computed from the wave spectra, and the surface wind field are imposed as time and space varying external point forcings to a LES model of the upper OBL. This one-way coupling allows us to examine how the OBL dynamics, including Langmuir turbulence, respond to rapidly varying large-scale forcings. This coupling strategy with LES neglects some large-scale processes, namely, Ekman pumping and mean-field advection, which are most important for the evolution of the spatially 3D hurricane wake at late times (Price 1981; Sanford et al. 2011).

Hurricane Frances was a category 4 hurricane that developed in the Atlantic basin in 2004 and was one of the most heavily studied storms in the Coupled Boundary Layer Air-Sea Transfer (CBLAST) program (Black et al. 2007). Novel profiling floats developed by Sanford et al. (2007), D'Asaro et al. (2007), and Sanford et al. (2011) document the upper-ocean mixing induced by Frances over several days including the development of the hurricane cold wake. The impact of the storm on the largescale currents and scalars is modeled by Zedler (2007) and Sanford et al. (2011).

\section{a. Winds and surface fluxes}

Surface winds, momentum, and heat flux parameterizations are needed to drive the LES and also the wave model. We make particular choices for our modeling but appreciate that there is uncertainty in specifying these external forcings owing to the variability in the available observations. An acceptable representation of the Hurricane Frances winds is derived from the NOAA hindcast data products described by Zedler (2007, see appendix). The specific formulas for the tangential and inflow radial winds $\left(v^{\phi}, v^{r}\right)$ expressed in terms of the radius $r$ are

$$
\begin{aligned}
& v^{\phi}(r)=53.86 e^{-0.0054 r}+2.86 \\
& v^{r}(r)=13.27 e^{-0.0035 r}-3.33,
\end{aligned}
$$

which apply over the domain $r_{\max } \leq r \leq 20 r_{\max }$, where $r_{\max }=40 \mathrm{~km}$ is the radius of maximum winds. As suggested by Zedler (2007), these expressions are augmented with a decaying exponential function for $r \leq$ $r_{\text {max }}$. In (1), the winds are in units of meters per second, $r$ is in units of kilometer, and the wind $v^{r}$ is positive inward. Based on the measurements of Sanford et al. (2007), we also add a constant translation speed $V_{H}=$ $5.5 \mathrm{~m} \mathrm{~s}^{-1}$ to (1), which results in an asymmetrical storm.

The parameterization of the surface stress $\tau$ at high winds is a key atmosphere-ocean coupling variable in hurricane prediction. There is vigorous debate concerning its parameterization but, as is customary, we adopt the bulk aerodynamic formula

$$
\boldsymbol{\tau}=\rho_{a} C_{d}\left|\mathbf{U}_{10}\right| \mathbf{U}_{10},
$$

where $\rho_{a}$ is the air density and $\mathbf{U}_{10}=\left(U_{10}, V_{10}\right)$ is the surface wind at a reference height $z_{a}=10 \mathrm{~m}$. The drag coefficient is parameterized with the Large and Pond (1981) prescription

$$
C_{d}= \begin{cases}0.0012, & \left|\mathbf{U}_{10}\right|<11 \mathrm{~m} \mathrm{~s}^{-1} \\ \left(0.49+0.065\left|\mathbf{U}_{10}\right|\right) \times 10^{-3}, & \left|\mathbf{U}_{10}\right|<25.0 \mathrm{~m} \mathrm{~s}^{-1} .\end{cases}
$$

This formula likely overpredicts the drag coefficient at very low winds $\left|\mathbf{U}_{10}\right| \sim 5 \mathrm{~m} \mathrm{~s}^{-1}$ but closely follows the observations at wind speeds $10 \leq\left|\mathbf{U}_{10}\right| \leq 20 \mathrm{~m} \mathrm{~s}^{-1}$. Based on a growing body of experimental data collected in the field and laboratory (Powell et al. 2003; Donelan et al. 2004; French et al. 2007) we further limit (saturate) the maximum $C_{d}=1.8 \times 10^{-3}$ at wind speeds beyond $20 \mathrm{~m} \mathrm{~s}^{-1}$. This saturated drag coefficient is near the estimates obtained from field observations but is lower than the value found by Donelan et al. (2004), $C_{d} \sim 2.3 \times 10^{-3}$, and Bell et al. (2012, see Fig. 19) who report a mean $C_{d} \sim 2.4 \times 10^{-3}$ above $50 \mathrm{~m} \mathrm{~s}^{-1}$ based on a budget analysis in the atmosphere. Sanford et al. (2011, see Fig. 7), using an integral momentum balance in the ocean, finds smaller values $C_{d} \sim 1.5 \times 10^{-3}$ in the wind speed range from 30 to $50 \mathrm{~m} \mathrm{~s}^{-1}$.

Surface latent $Q_{\text {lat }}$ and sensible $Q_{\text {sen }}$ fluxes are also imposed at the water surface in the LES. The parameterization of these fluxes is

$$
\begin{gathered}
Q_{\text {sen }}=\rho_{a} C_{h} \mathcal{C}_{p}\left|\mathbf{U}_{10}\right| \Delta T, \\
Q_{\text {lat }}=\rho_{a} C_{e} L v\left|\mathbf{U}_{10}\right| \Delta q,
\end{gathered}
$$

where $\mathcal{C}_{p}$ is the specific heat, $L v$ is the latent heat of vaporization, $(\Delta T, \Delta q)$ are the temperature and specific humidity differences between the sea surface and the reference height, and $\left(C_{h}, C_{e}\right)$ are exchange coefficients. We choose $C_{h}=C_{e}=1.5 \times 10^{-3}, \Delta T=2.5 \mathrm{~K}$, and compute $\Delta q$ based on a relative humidity of $80 \%$ at the reference height and assuming saturation at the sea surface. These choices are representative values used in hurricane models (e.g., Price 1981; Jacob et al. 2000; Jacob and Shay 2003; D'Asaro 2003; Zedler et al. 2009) and result in a total heat flux from the ocean to the atmosphere of more than $1000 \mathrm{~W} \mathrm{~m}^{-2}$ at the time of maximum winds. Despite this seemingly high value the surface heat flux is $\sim 0.1$ of the dominant entrainment flux at the time of maximum winds. The sensitivity of the ocean boundary layer evolution to the surface exchange coefficients is described by Zedler et al. (2009). 


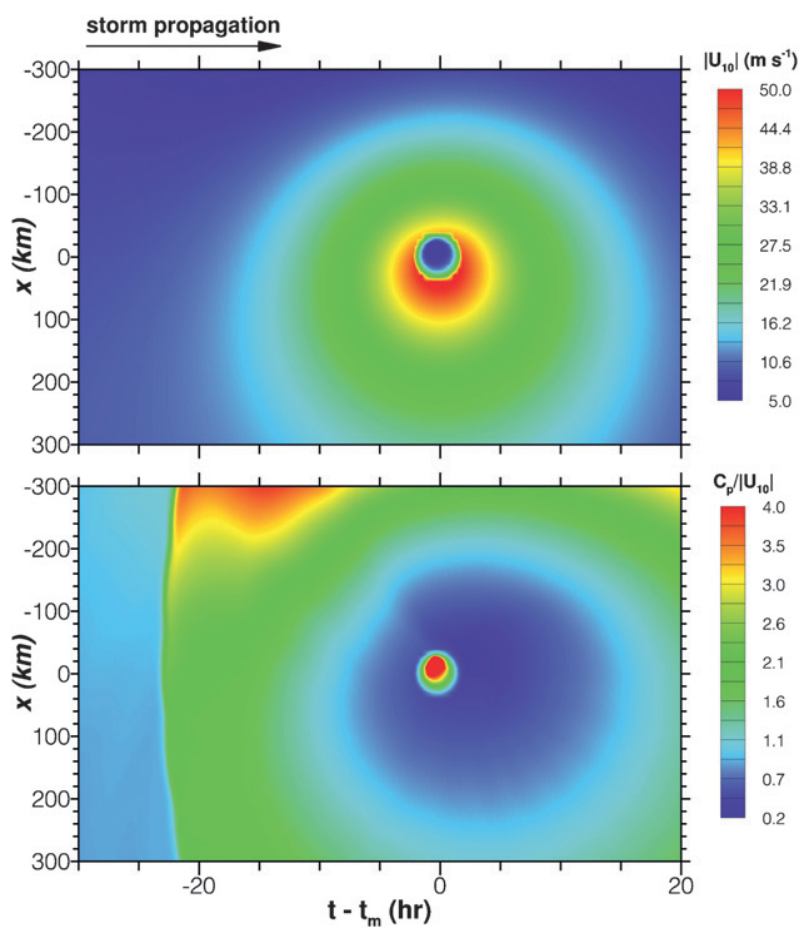

FIG. 1. The hurricane storm propagates from left to right, and the fields are shown in the form of an $X-t$ Hovmöller diagram; that is, the temporal variation of a particular field is shown for a chosen $X$ cross section. Time is referenced to the time of maximum winds $t_{m}$. Wind speed $\left|\mathbf{U}_{10}\right|$ (color scale $\mathrm{m} \mathrm{s}^{-1}$ ) imposed in the WaveWatch III simulations and (bottom) the wave age $A=C_{p} /\left|\mathbf{U}_{10}\right|$ over the lifetime of the storm.

\section{b. Wave field}

The surface wind and wave fields input to the LES are products derived from the wave prediction model WaveWatch III (Tolman 2002) adapted to hurricane conditions (Romero and Melville 2010b; L. Romero et al. 2012, manuscript in preparation). The hurricane wave simulations use nearly exact computations of the nonlinear energy transfers (van Vledder 2006), wind input by Snyder et al. (1981), and the energy dissipation by Romero and Melville (2010b) with the following modifications. For this study, the dissipation is improved to allow a smoother development of the spectrum with multiple peaks and to allow the spectral tail to follow the rapidly turning winds, while matching the empirical degree of saturation with weak dependence on the wind speed or friction velocity (Banner et al. 1989; Romero and Melville 2010a). A description of the code and its validation for high winds and hurricanes is given by Romero and Melville (2010b) and Romero et al. (2012).

For the present application, the wind-wave simulations of Hurricane Frances are carried out in an $X-Y$ domain $( \pm 410, \pm 810) \mathrm{km}$ with a horizontal resolution of

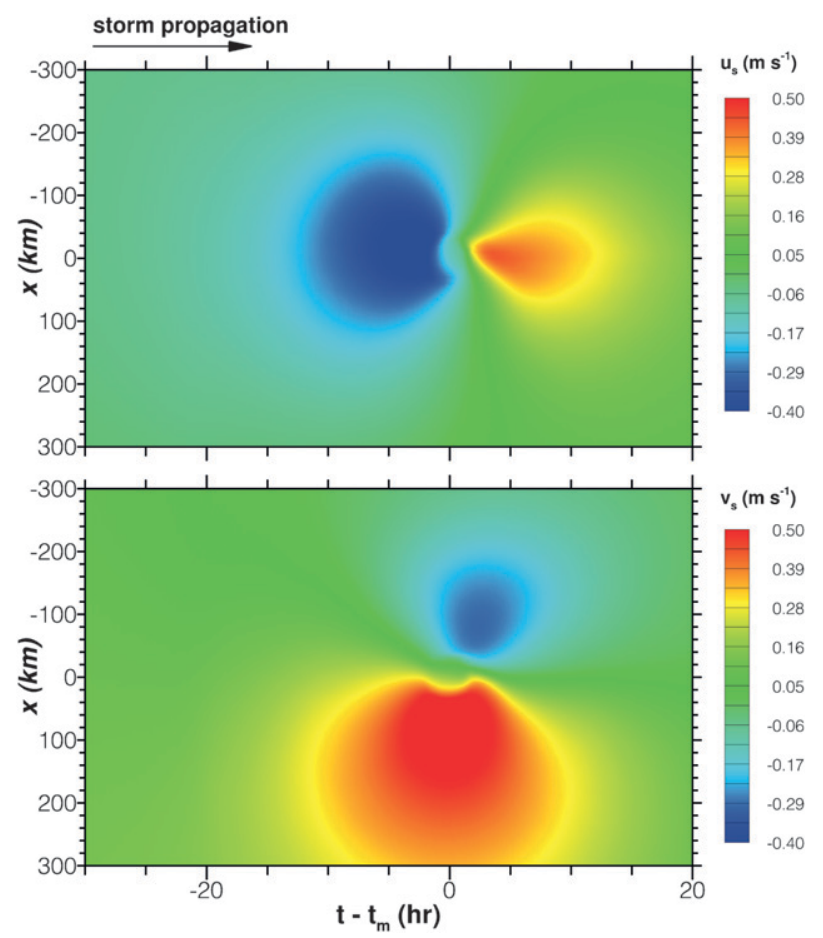

FIG. 2. Stokes velocity components (top) $u_{s}$ and (bottom) $v_{s}$ at the water surface for the imposed wind field (color scale units $\mathrm{m} \mathrm{s}^{-1}$ ).

$10 \mathrm{~km}$, that is, $83 \times 163$ grid points. The spectral grid is discretized with a directional resolution of $10^{\circ}$ using a constant relative bandwidth $d \varpi / \varpi=0.078 ; \varpi$ is the radial frequency. The range of resolved frequencies is $[0.157,15.341] \mathrm{rad} \mathrm{s}^{-1}$ corresponding to the wavenumber range $[0.0025,24] \mathrm{rad} \mathrm{m}^{-1}$. The storm eye is initially placed at $(X, Y)=(0,-510) \mathrm{km}$ and is translated in time. Time series of winds and waves are collected at 61 discrete points distributed along the line $(X, Y)=( \pm 300,0)$ $\mathrm{km}$. The primary output of the wave model is the $2 \mathrm{D}$ wave height spectrum $F(\varpi, \phi)$, which varies with $\varpi$ and angular position $\phi$. The wave spectra are postprocessed to generate the Stokes drift velocity and wave age fields.

The $X-t$ Hovmöller contour maps presented in Figs. 1 and 2 illustrate some of the variability in the wind and wave fields induced by our hypothetical storm over the horizontal domain $X= \pm 300 \mathrm{~km}$ for a time period of more than $50 \mathrm{~h}$. In these and subsequent figures, time is referenced to the time of maximum winds $t_{m}$ on the right-hand side of the storm. Here $t_{m}=45.625 \mathrm{~h}$ is based on a constant storm translation speed $V_{h}=5.5 \mathrm{~m} \mathrm{~s}^{-1}$ and the initial storm center location. Thus, the cross section $(X, Y=0)$ is ahead of the storm for $t-t_{m}<0$, cuts through the storm eye at $t-t_{m}=0$, and is behind the storm (in the storm wake) for $t-t_{m}>0$. Inspection of the contours in Fig. 1 shows that the wind speed $\left|\mathbf{U}_{10}\right|$ is asymmetrical about $X=0 \mathrm{~km}$, a consequence of the 
translation speed $V_{h}$, with the largest values $\sim 50 \mathrm{~m} \mathrm{~s}^{-1}$ on the right-hand side of the storm track.

Wave age, shown in the lower panel of Fig. 1, is defined as $A=C_{p} /\left|\mathbf{U}_{10}\right|$ where $C_{p}$ is the phase speed of the peak in the wave height spectrum: $A$ varies widely in space and time across the storm path. Extensive periods of growing waves $A \sim 0.5$ are observed near the time of maximum winds with the lowest wave age on the righthand side of the storm. For time periods well ahead of the storm center, $A \sim 1.2$ indicative of near wind-wave equilibrium conditions. At $t-t_{m} \sim-22 \mathrm{~h}$, the wave age jumps abruptly, $\mathrm{A}>2$, as fast-moving swell propagates rapidly ahead of the storm. In the wake of the storm $t-$ $t_{m}>0$, the wave age again slowly approaches wind wave equilibrium and at long times $t-t_{m}>20 \mathrm{~h}$ the wave state again becomes swell dominated. These estimates of wave age in a hurricane are consistent with values deduced from observations of 1D spectra documented by Young $(1998,2006)$ who reports values ranging from $[0.5-2.0]$.

A key variable for our LES is the Stokes drift velocity $\mathbf{u}_{s}$, which appears in the vortex force $\mathbf{u}_{s} \times \overline{\boldsymbol{\omega}}$ where $\overline{\boldsymbol{\omega}}$ is the resolved vorticity; see (8). For a broadband wave spectrum, $\mathbf{u}_{s}$ is obtained by the integration (Kenyon 1969):

$$
\mathbf{u}_{s}(z)=2 \int_{0}^{\infty} \int_{-\pi}^{\pi} \mathbf{k} \varpi F(\varpi, \phi) e^{2|\mathbf{k}| z} d \phi d \varpi,
$$

where $\mathbf{k}$ denotes the horizontal wavenumber vector [also see McWilliams and Restrepo (1999)]. The Stokes drift velocity $\mathbf{u}_{s}$ inherits the complexity of the wave field through (5); it varies with wind speed, spatial position, and time as depicted in Fig. 2. For our particular choice of storm propagation direction, the largest Stokes drift occurs in component $v_{s}$ on the right-hand side of the storm track. The distribution of the wave field is more asymmetrical than the wind field mainly due to the "extended" fetch on the right-hand side of a translating hurricane (Young 2003). The asymmetrical distribution of $v_{s}$ about $X=0 \mathrm{~km}$ reflects the stronger winds and better wind wave alignment on the storm right hand side. Clearly $u_{s}$ is largest (most negative) in front of the storm center with contours roughly symmetrical about $X=0 \mathrm{~km}$.

McWilliams et al. (1997) introduced a surface based turbulent Langmuir number

$$
\mathrm{La}_{t}=\sqrt{\frac{u_{*}(X, t)}{\left|\mathbf{u}_{s}(X, z=0, t)\right|}}
$$

as a measure of the relative importance of shear forcing to vortex force. In (6) the water friction velocity $u_{*}(X, t)=\sqrt{|\boldsymbol{\tau}| / \rho_{w}}$, where $\rho_{w}$ is the density of water, $\mathbf{u}_{s}$ is evaluated at the water surface, and $\boldsymbol{\tau}$ is given by (2). Grant and Belcher (2009) also adopt (6) in their scaling
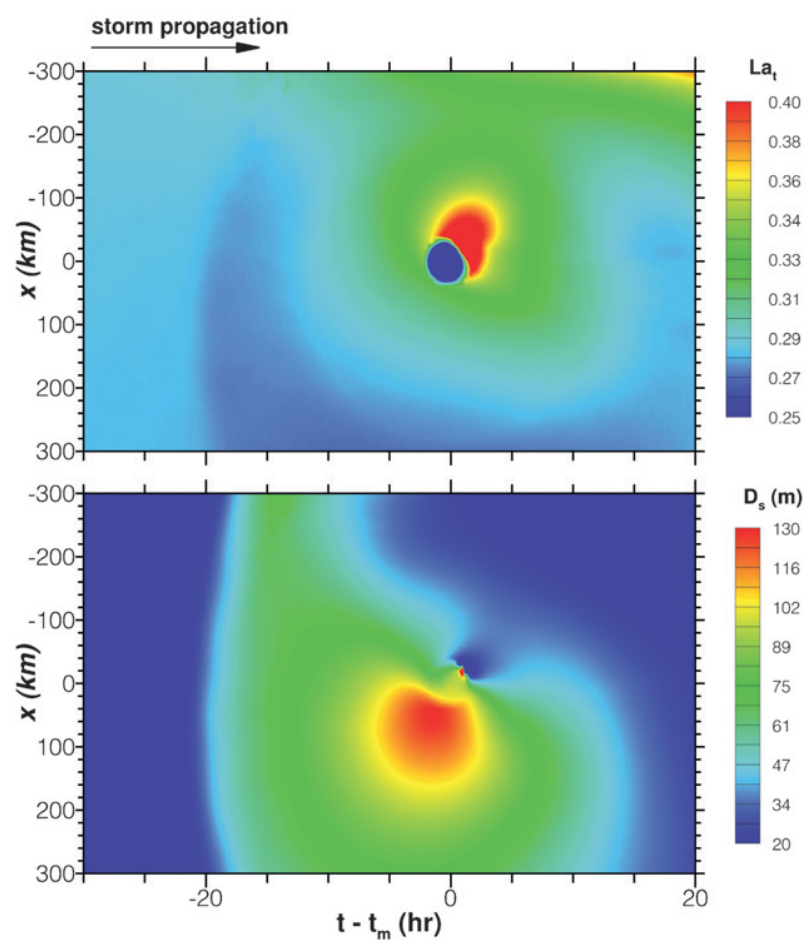

FIG. 3. (top) The turbulent Langmuir number $\mathrm{La}_{t}$ (nondimensional color scale) and (bottom) Stokes depth scale $D_{s}$. In the bottom panel the color bar is in units of meters.

of the turbulent kinetic energy equation with wave effects. Harcourt and D'Asaro (2008) prefer a surface based Langmuir number LasL $=\sqrt{u_{*} /\left\langle u_{s}\right\rangle_{\mathrm{SL}}}$, where $\left\langle u_{s}\right\rangle_{\mathrm{SL}}$ is the average Stokes drift in the surface layer. These definitions of Langmuir number are, however, strictly only applicable in the situation of aligned winds and waves; Van Roekle et al. (2012) attempts to generalize (6) for wind-wave misalignment. Here we adopt (6) as our working definition of Langmuir number because of its simplicity and the ability to compare with previous studies; note that in (6) $\mathbf{u}_{s}$ is computed from the full wave spectrum according to (5).

Figure 3 shows contours of turbulent Langmuir number for our hypothetical storm. Outside the radius of maximum winds there are broad regions where $0.25<$ $\mathrm{La}_{t}<0.4$. The very low values in the storm eye should be favorable for generating Langmuir cells with high aspect ratio, i.e., with their streamwise-to-crosswise length scale much greater than unity (Chini et al. 2009). Historically the criterion $\mathrm{La}_{t}<0.4$ (e.g., $\mathrm{Li}$ et al. 2005), used for aligned winds and waves, implies that often the hurricane driven OBL should be in a regime favorable to the generation of Langmuir turbulence. However, the applicability of the criterion $\mathrm{La}_{t}<0.4$ for nonequilibrium situations where weaker Langmuir cells can develop offaxis between the wind and wave directions is unknown (Van Roekle et al. 2012). 
The vertical profile of Stokes drift $\mathbf{u}_{s}(z)$ spirals with depth, a consequence of a multicomponent wave field composed of swell and wind waves that propagate in different directions. To characterize the depth penetration by the Stokes drift, an important variable in Langmuir turbulence, we define a vertical length scale

$$
D_{s}=4 \pi \int_{z_{s}}^{0} \frac{\mathbf{u}_{s}(z, t) \cdot \mathbf{u}_{s}(0, t)}{\left|\mathbf{u}_{s}(0, t)\right|^{2}} d z,
$$

where the integral is taken over the interval $z_{s}<z<0$ with $z_{s}$ chosen as the depth where the integrand remains positive [see Harcourt and D'Asaro (2008) for an alternate length scale for aligned winds and waves]. The above definition is robust and reduces to $D_{s}=\lambda=2 \pi / k$ for a monochromatic wave field. The variation of $D_{s}(X, t)$ is given in Fig. 3. Over the time interval $(-20,10) \mathrm{h}, D_{s} \sim$ $60 \mathrm{~m}$ with values greater than $130 \mathrm{~m}$ on the right-hand side of the storm at the time of maximum winds. At $t-t_{m} \sim$ $5 \mathrm{~h}$, there is a small region to the left of the storm eye where $D_{s}$ becomes small, a reflection of the confused sea state induced by opposing winds and waves. This is also a region where $\mathrm{La}_{t}>0$.6. Based on the results in Fig. 3 we conclude that the vertical profile of Stokes drift is track dependent and differs noticeably from a monochromatic prescription, but occupies a significant fraction of the OBL even at the time of maximum winds; that is, $D_{s} /|h|>1$ where $h$ is the OBL depth.

\section{LES problem posing}

The structure of the hurricane driven OBL depends on the large-scale forcings discussed in section 2. To thoroughly investigate all possible combinations of wind and wave forcing is beyond our computational reach with the LES and, thus, we restrict our attention to two locations $X= \pm 60 \mathrm{~km}$ from the storm center shown in Fig. 1. These locations are of interest because they feature high wind conditions with different wind stress time histories and have a mixture of differently oriented winds and waves. As a further parameter variation, on each side of the storm, simulations are also conducted with and without surface wave effects, that is, with and without Stokes drift which generates the vortex force.

\section{a. LES model}

The details of our LES model for the OBL with wave effects are extensively discussed by McWilliams et al. (1997) and Sullivan et al. (2007). The equations for the resolved flow components are the Craik-Leibovich (CL) theory with crucial wave-current coupling through the vortex force. The LES model, with wave effects, is based on the incompressible Boussinesq equations with a single- point, second-moment turbulent kinetic energy (TKE) closure subgrid-scale parameterization and a flat ocean surface (Moeng 1984; McWilliams et al. 1997). The added wave effects are the vortex force, Stokes-Coriolis coupling, Lagrangian mean advection associated with Stokes drift, a wave-averaged increment to the pressure that arises through conservative wave-current interaction (McWilliams et al. 1997), and Stokes production in the subgrid-scale (SGS) TKE equation (Sullivan et al. 2007). Additional acceleration and energy generation due to nonconservative stochastic wave breaking, described by Sullivan et al. $(2004,2007)$, are not considered here.

We use standard LES notation where spatially filtered (or resolved) variables are denoted with an overbar: $(\overline{\mathbf{u}}, \overline{\boldsymbol{\omega}}=\boldsymbol{\nabla} \times \overline{\mathbf{u}})$ are the resolved-scale velocity and vorticity; $e$ is the subgrid-scale TKE; $\bar{\theta}$ is the temperature; and $\pi$ is a generalized pressure variable. Other variables appearing in the LES equation set are the Coriolis frequency $\mathbf{f}=(0,0, f)$; reference temperature $\widetilde{\theta}_{o} ;$ gravitational acceleration $g$; and subgrid-scale momentum and density fluxes, $\left(\tau_{i j}, \tau_{i \theta}\right)$, respectively. These subgrid-scale fluxes are modeled using the eddy viscosity prescription described in Moeng (1984) and Sullivan et al. (1994), which implies that the principal feedback of $e$ on $\overline{\mathbf{u}}$ and $\bar{\theta}$ occurs through subgrid-scale mixing with eddy viscosity and diffusivities $\left(\nu_{t}, \nu_{s}\right) \propto e^{1 / 2}$.

In the situation of misaligned winds and waves, Stokes drift velocity couples to the resolved vorticity $\overline{\boldsymbol{\omega}}=(\xi, \eta, \zeta)$ to generate a three-dimensional vortex force

$$
\mathbf{u}_{s} \times \overline{\boldsymbol{\omega}}=\left(v_{s} \zeta,-u_{s} \zeta, u_{s} \eta-v_{s} \xi\right)
$$

that contributes to all three resolved momentum equations; in the case of aligned winds and waves, which is most often studied, the vortex force reduces to the twocomponent form $u_{s}(0,-\zeta, \eta)$.

\section{b. LES setup}

Two computational domains of varying size are used in the LES. First, the vertical dimension of the computational box is chosen to be about twice the expected maximum depth of the OBL; based on observations $|h|_{\max } \sim 120 \mathrm{~m}$ (Sanford et al. 2011) and thus we choose a box of depth $-240 \mathrm{~m}$. The horizontal dimension is picked to be $\sim 5|h|_{\max }$ so as to allow the interior motions to develop independent of the periodic sidewall boundary conditions (see Schmidt and Schumann 1989). On the lefthand side $X=-60 \mathrm{~km}$, which has slightly weaker forcing, a small domain $\left(L_{x}, L_{y}, L_{z}\right)=(750,750,-240) \mathrm{m}$ was deemed acceptable. On the right-hand side of the storm, $X=60 \mathrm{~km}$, the vigorous forcing from the inertially rotating wind stress was found to generate a system of large-scale random gravity waves in the hurricane wake. 
Test runs indicated that these structures were only adequately captured by increasing the horizontal area of the small domain LES by a factor of 4 . Thus, a large domain is used on the right hand side with $\left(L_{x}, L_{y}, L_{z}\right)=$ $(1500,1500,-240) \mathrm{m}$. The number of grid points in the small domain is $\left(N_{x}, N_{y}, N_{z}\right)=(512,512,256)$ and is increased to $(1024,1024,256)$ in the large domain; thus the horizontal spacing $\Delta x=\Delta y=1.46 \mathrm{~m}$ in all the computational boxes. In the vertical direction, we use a stretched vertical grid where the spacing between any neighboring cells is held fixed at the ratio $K=1.00451$ with the first $w$ grid point located at $\Delta z_{1}=-0.5 \mathrm{~m}$; thus the first $u$ point is at $z_{1} / 2=-0.25 \mathrm{~m}$ in a staggered grid. Based on these choices, the cell aspect ratio $\Delta x / \Delta z=$ $(2.93,1.54)$ at $z=(0,-100) \mathrm{m}$. The small and large computational boxes are able to capture small-scale turbulence at early times and larger scales of motion that develop during the rapid deepening of the OBL at the time of maximum winds.

The initial temperature sounding is simply constructed from two linear segments: over the depth $h_{i} \leq$ $z \leq 0, \theta_{i}=302.4 \mathrm{~K}$ and for $z<h_{i}$ the temperature decreases at a rate of $0.04 \mathrm{~K} \mathrm{~m}^{-1}$. This is approximately the variation observed for Hurricane Frances (Sanford et al. 2011). The depth $h_{i}=-32 \mathrm{~m}$ is the initial OBL depth. The Coriolis parameter is $f=0.681 \times 10^{-4} \mathrm{~s}^{-1}$, corresponding to an inertial period $T=25.6 \mathrm{~h}$. The simulations are initiated from rest with small random temperature perturbations under the wind and wave forcing from hour 10 of the WaveWatch III simulation. At this time, the wind and wave fields are slowly varying with winds less than $10 \mathrm{~m} \mathrm{~s}^{-1}$. Next, these external forcings are held constant and the simulations are integrated for 10 physical hours to generate fully developed equilibrium turbulence. The resulting currents are $\sim 0.05 \mathrm{~m} \mathrm{~s}^{-1}$, and the initial state of these weak currents has minimal impact on the long time ocean response under strong forcing (Crawford and Large 1996, p. 889). Finally, the winds and Stokes drift are allowed to follow the time history shown in Figs. 5 and 6. The time step $\Delta t$ in the code is dynamically picked to obey a fixed CourantFredrichs-Lewy condition. Here $\Delta t$ varies by an order of magnitude, from 0.3 to $5 \mathrm{~s}$, and more than 300000 time steps are needed to cover the 70 physical hours of the simulation. With a storm translation speed of $5.5 \mathrm{~m} \mathrm{~s}^{-1}$ the LES domains are initially located more than $900 \mathrm{~km}$ from the storm center. The parallelization of the code is described by Sullivan and Patton (2011).

\section{Transient wind and wave forcing applied to LES}

The temporal and spatial variability in the large-scale forcing conditions critically impacts the time evolution

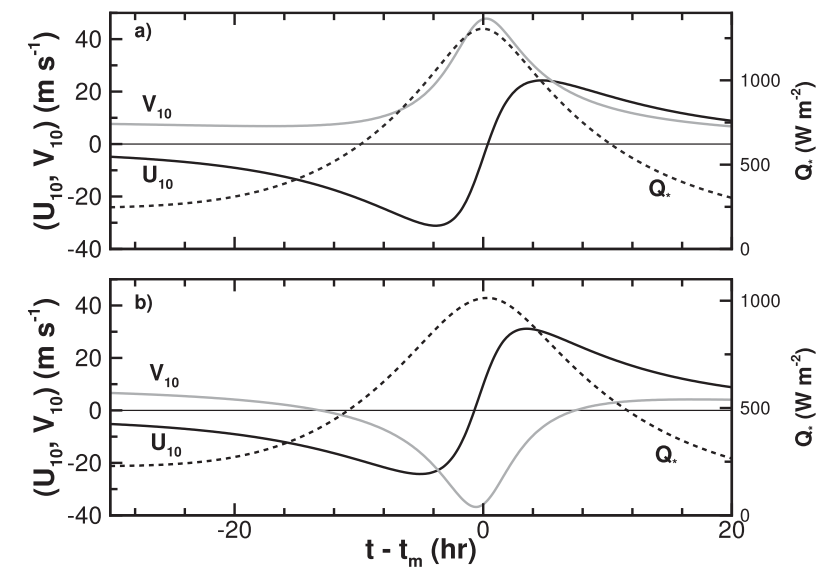

FIG. 4. External winds $\left(U_{10}, V_{10}\right)$ and cooling $Q_{*}$ applied to the LES: (a) right-hand side of the storm $X=60 \mathrm{~km}$ and (b) left-hand side of the storm $X=-60 \mathrm{~km}$.

of the OBL and the development of Langmuir turbulence. To aid the interpretation of the OBL dynamics generated by the simulations we first discuss the time history of winds, waves, and cooling that are applied at the upper boundary of the LES; see Figs. 4, 5, 6, and 7. In these figures, the winds $\mathbf{U}_{10}$, surface cooling flux $Q_{*}=$ $Q_{\text {sen }}+Q_{\text {lat }}$, Stokes drift velocity $\mathbf{u}_{s}$, wind-wave alignment, and surface wind stress $\tau$ are time slices at the horizontal locations $X= \pm 60 \mathrm{~km}$ taken from Figs. 1 and 2.

The primary forcing to the LES (and also the wave model) are the surface winds, shown in Fig. 4, converted to a surface wind stress according to the prescription (2). The amplitude of the wind stress, depicted in Figs. 5 and 6 , is a strong function of $(X, t)$ within the storm track and, as expected, each component displays a strong pulse at the time of maximum winds mimicking the surface winds. Figures 5 and 6 also compare the time varying stress $\boldsymbol{\tau}(t)$ with an "inertial" stress of equal magnitude that rotates clockwise with frequency $f$; that is, $\boldsymbol{\tau}_{i}(t)=|\boldsymbol{\tau}(t)| e^{-i f t}$. This exposes the direction and rate of rotation relative to an inertial motion. Both wind stress components on the right side of the storm track are partially resonant with the ideal inertial stress $\boldsymbol{\tau}_{i}$. The period of resonance is relatively short, about $10 \mathrm{~h}$, but occurs during the time of maximum winds $(-5,5) \mathrm{h}$. Meanwhile, on the left-hand side, the wind stress magnitudes are modestly lower than on the right-hand side and component $\tau_{y}$ clearly rotates in a direction opposite to that of an inertial rotation. Transient wind forcing at frequency $f$ with clockwise rotation is able to drive currents below the mixed layer and thereby efficiently mix the OBL (e.g., Price 1981; Crawford and Large 1996; Skyllingstad et al. 1999). The observations of Sanford et al. (2011) demonstrate how wind stress with limited resonance time is able to efficiently mix a hurricane OBL. At early and, more so, at late times the 

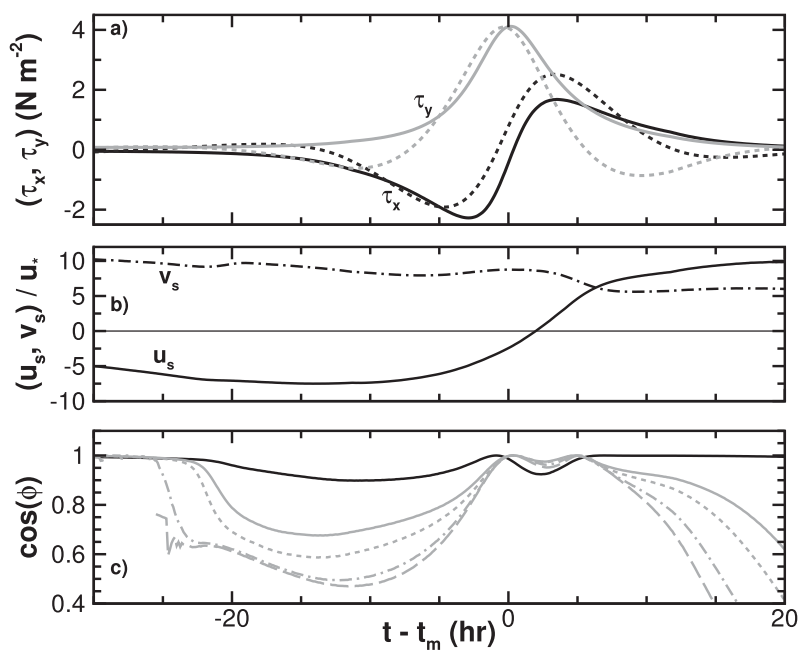

FIG. 5. Surface wind stress and wave forcing on the right hand side of the storm, $X=60 \mathrm{~km}$ : (a) time variation of the wind stress $\tau$ where the (black, gray) lines are the components $\tau=\left(\tau_{x}, \tau_{y}\right)$, respectively. The (black, gray) dotted lines are resonant stress components $\tau_{i}(t)$ that rotate clockwise with inertial frequency $f$; that is, $\boldsymbol{\tau}_{i}(t)=|\boldsymbol{\tau}(t)| e^{-i f t}$. (b) The surface Stokes drift velocity components $\left(u_{s}, v_{s}\right)$ normalized by the local friction velocity $u_{*}(X, t)$ are depicted. (c) The wind-wave alignment given by (9) at the water surface (black line) and at depth $z=-(5.8,12.4,40,80.2) \mathrm{m}$ gray (solid, dotted, dash-dot, long dash) lines, respectively.

wind stress rotation applied at the upper boundary of the LES is noninertial on both sides of the storm track.

Surface cooling plays a minor role, despite the more than $1200 \mathrm{~W} \mathrm{~m}^{-2}$ applied at the time of maximum winds; it is offset by the rapid increase in surface shear stress. The ratio $\left|h_{i}\right| / L$ where $L=-u_{*}^{2} / \beta \kappa Q_{*}$ is the MoninObukhov length is often used in atmospheric boundary layer analysis as a measure of the relative importance of shear and buoyancy forcing at the surface (e.g., Deardorff 1972; Moeng and Sullivan 1994). In this expression, the buoyancy parameter $\beta=g / \theta_{o}$ and $\kappa=0.4$ is the von Kármán constant. Broadly, over the bulk of the time period the OBL surface shear forcing dominates surface cooling since $-1<\left|h_{i}\right| / L<0$ and during the time period of maximum winds, $(-10,10) \mathrm{h}$, we find $-0.1<\left|h_{i}\right| / L<0$. Cooling plays a modest role at early times and contributes to the well-mixed (vertically uniform) shape of the mean current profiles.

It is informative to discuss how the Stokes velocity varies in our simulations since it impacts the development of Langmuir turbulence. In the lower and middle panels of Figs. 5 and 6 we show the time variation of the windStokes alignment

$$
\cos \phi=\frac{\mathbf{U}_{10}(t) \cdot \mathbf{u}_{s}(z, t)}{\left|\mathbf{U}_{10}(t)\right|\left|\mathbf{u}_{s}(z, t)\right|}
$$
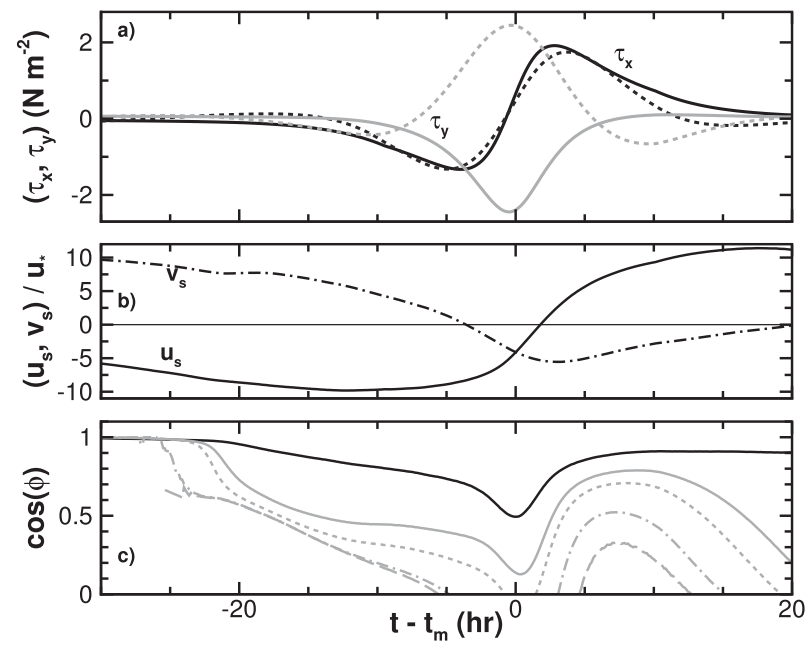

FIG. 6. Surface wind stress and wave forcing on the left-hand side of the storm, $X=-60 \mathrm{~km}$. The panels, labeling and variables are the same as in Fig. 5. Note the difference in the vertical scale in the lower panels of Figs. 6 and 5.

at depths $z=(0,-5.8,-12.4,-40,-80.7) \mathrm{m}$, and the components of the normalized Stokes drift velocity at the surface $\left(u_{s}, v_{s}\right) / u_{*}$. The surface based $\mathrm{La}_{t}$, given by (6), can be inferred from this plot and its range is narrow; along $X=60 \mathrm{~km}, 0.28<\mathrm{La}_{t}<0.32$ and along $X=$ $-60 \mathrm{~km}, 0.28<\mathrm{La}_{t}<0.41$. First, we notice $u_{s}(0, t) / u_{*}$ has the same shape and amplitude on both sides of the storm track, $X= \pm 60 \mathrm{~km}$. This is expected based on the contours of Stokes drift velocity in Fig. 2. Thus, the important differences in $\mathbf{u}_{s} / u_{*}$ between the right-hand and left-hand sides are due to component $v_{s} / u_{*}$. On the right-hand side of the track $v_{s}(0, t) / u_{*}$ is nearly constant with time, less than a $25 \%$ variation over the period $(-30,5) \mathrm{h}$. This correlation between Stokes drift and wind stress is meaningful since $u_{*}$ varies by a factor of 7 over the time period of the simulations. Meanwhile on the left-hand side $v_{s}(0, t) / u_{*}$ at first decreases linearly, then changes sign and finally reaches a minimum near $t-t_{m}=0 \mathrm{~h}$. These differences are a result of the extended fetch on the right side of the storm versus the progressive misalignment between winds and waves on the left side as time advances.

The wind-Stokes alignment is higher at the surface than at depth, which results from a multimode surface wave spectrum composed of wind waves and swell: recall in wind wave equilibrium $\cos \phi=1$ at all $z$. Short wavelength wind waves rapidly align themselves to varying surface shear forcing compared to the slow adjustment of long wavelength swell; this pattern of wave development in a hurricane is reported by Wright et al. (2001). Thus surface values of Stokes drift are only a partial reflection of the wave state that drives Langmuir 

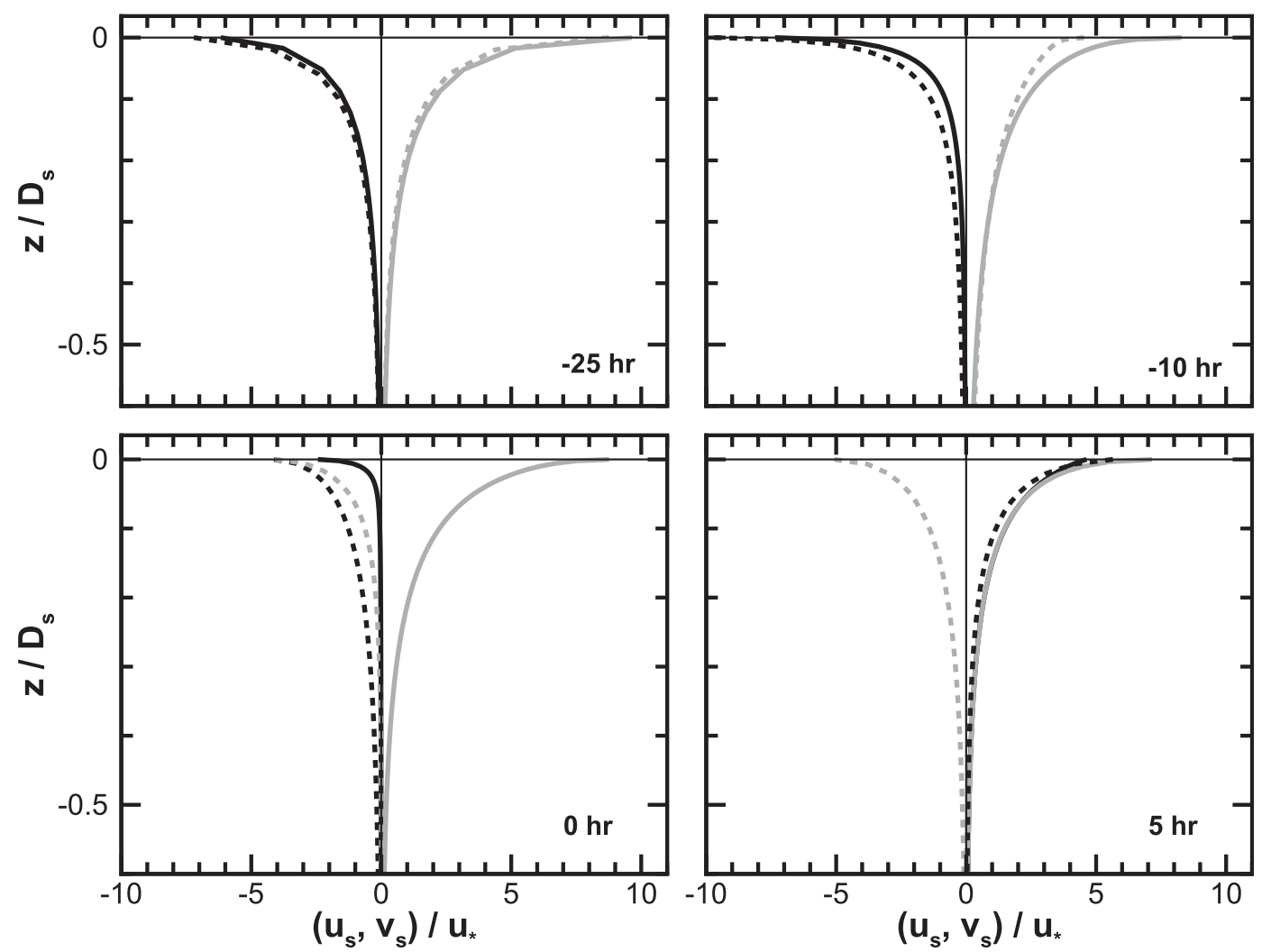

FIG. 7. Vertical profiles of Stokes drift velocity normalized by the local friction velocity $\left(u_{s}, v_{s}\right) / u_{*}$ at time instances $t-t_{m}=(-25,-10,0,5) \mathrm{h}: z$ is made dimensionless by the Stokes depth scale $D_{s}$ given by (7). The solid (black, gray) lines are $\left(u_{s}, v_{s}\right) / u_{*}$, respectively, along the line $X=60 \mathrm{~km}$. The dashed (black, gray) lines are their counterparts along the line $X=-60 \mathrm{~km}$. For the selected time instances the depth scale $D_{s}=(14.5,86.0,123.5,74.7) \mathrm{m}$ and $(12.6$, $75.2,45.7,37.1) \mathrm{m}$ for $X=60(-60) \mathrm{km}$. Note at $t-t_{m}=5 \mathrm{~h}$ the solid black and gray lines overlap.

turbulence. Swell first appears at $t-t_{m}=-22 \mathrm{~h}$, as indicated by wave age $A>1.2$ in the lower panel of Fig. 1 , and reduces the wind-Stokes alignment at depth throughout the passage of the storm. At the surface, on the righthand side of the storm the wind-Stokes alignment is always within $10^{\circ}$ at the surface and no more than $45^{\circ}$ at $z=-5.8 \mathrm{~m}$. At the time of maximum winds the Stokes drift is near "resonant" with the winds; that is, the windStokes alignment is near unity over a depth of more than $80 \mathrm{~m}$ and the Stokes depth scale exceeds $130 \mathrm{~m}$. Thus we anticipate strong Langmuir turbulence to develop at this time. Over the time period $(-5,5) \mathrm{h}$, the wind-Stokes alignment on the left-hand side increases, $60^{\circ}$ at the surface and almost $90^{\circ}$ at $z=-10.2 \mathrm{~m}$, because the wind and dominant wave propagation directions are opposed.

Selected vertical profiles of the Stokes drift velocity are shown in Fig. 7 at times before, at, and after the time of maximum winds. These profiles illustrate that the normalized surface values of $\mathbf{u}_{s} / u_{*}$ and, hence, $\mathrm{La}_{t}$, have only a mild variation over the time period. The invariance of $\mathrm{La}_{t}$ however disguises the profile shape changes that occur with $(X, t)$. For example, the profiles of $v_{s}(z, t)$ at $t-t_{m}=(-25,0) \mathrm{h}$ have similar surface values of $\mathrm{La}_{t}$ but the profile at $t-t_{m}=0$ extends much deeper into the OBL: at $t-t_{m}=(-25,0,0) \mathrm{h} D_{s}=(14.5,123) \mathrm{m}$, respectively. Intuitively, we expect the formation of Langmuir turbulence to depend on $\mathrm{La}_{t}$, the vertical shape of the Stokes profile and its depth penetration.

\section{Identification of Langmuir turbulence}

\section{a. Temporal evolution of vertical velocity}

The LES of steady aligned wind wave flows and a few field observations find vertical velocity is a robust signature of Langmuir turbulence. In the Langmuir turbulence regime, coherent Langmuir cells form and these structures can be detected in the instantaneous vertical velocity field and furthermore impact the statistical moments of $w$. Figure 8 compares the maximum total (resolved plus SGS) second-order moment $\left\langle w^{2}\right\rangle_{\max }$ and the minimum resolved third-order moment $\left\langle\bar{w}^{3}\right\rangle_{\min }$ diagnosed from our simulations. Since the LES equations are closed at the second-moment level there is no 

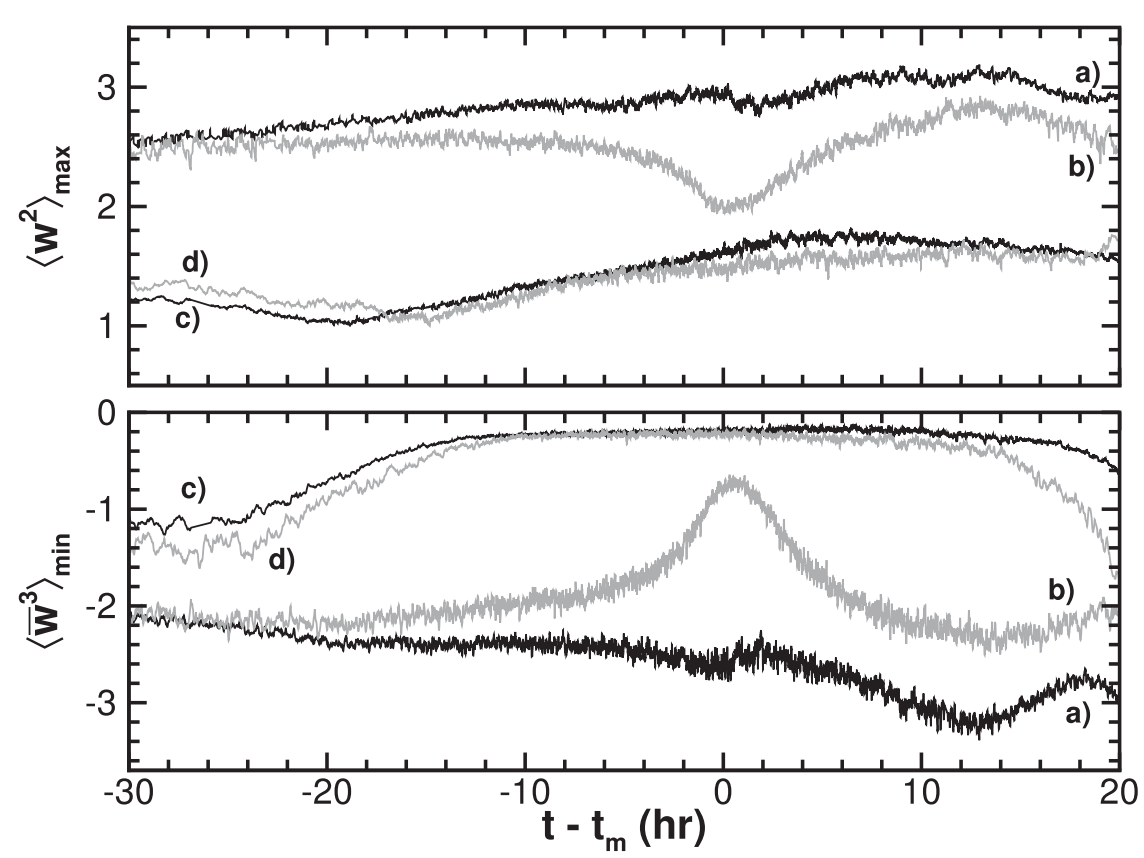

FIG. 8. Temporal variation of the maximum total vertical velocity variance $\left\langle w^{2}\right\rangle_{\max }$ and minimum resolved third-order moment $\left\langle\bar{w}^{3}\right\rangle_{\min }$ for cases with and without wave effects at locations left and right of the storm center. Simulations with wave effects for (a) $X=60 \mathrm{~km}$ and (b) $X=-60 \mathrm{~km}$ and simulations with no wave effects for (c) $X=60 \mathrm{~km}$ and (d) $X=-60 \mathrm{~km}$. The moments are normalized appropriately by either $u_{*}^{2}(X, t)$ or $u_{*}^{3}(X, t)$.

estimate of third-order SGS quantities. Both moments are normalized by local $u_{*}(X, t)$. These temporally varying moments are obtained by first computing the vertical profiles of resolved $\left.\langle\overline{w(z, t)})^{2}\right\rangle$ and $\left\langle\overline{w(z, t)}^{3}\right\rangle$ at all $z$ for a given $t$ : angle brackets denote an $x-y$ spatial average over the LES domain. The $\left\langle\overline{w(t)^{2}}\right\rangle_{\max }$ and $\langle\overline{w(t)}\rangle_{\min }$ are found by searching for the appropriate maximum or minimum along their respective vertical profiles. We then add an estimate of the SGS contribution, $2 e / 3$, to the resolved $w$ variance; that is, $\left\langle w^{2}\right\rangle_{\max }$ is the sum of resolved and SGS contributions. We note that the vertical locations of $\left\langle w^{2}\right\rangle_{\max }$ and $\left\langle\bar{w}^{3}\right\rangle_{\min }$ are not the same and vary with time. These positions are always located in the upper surface layer of the OBL but are found deeper in the water as the winds and waves increase.

The time series depicted in Fig. 8 are compelling evidence for Langmuir turbulence in the hurricane driven OBL. The $w$ moments are sensitive to the location within the storm track, depend on the time and space evolving wind and wave fields, and the inclusion of phase averaged wave-current interactions in the LES. Over the lifetime of the vortex-force simulation $\left\langle w^{2}\right\rangle_{\max }>2.5$ along the track $X=60 \mathrm{~km}$. Notice also that during the time period with resonant wind forcing, $(-5,5) \mathrm{h}$, the maximum vertical velocity variance is near 3 and this level persists in the hurricane wake. Recall $u_{*}$ varies by about a factor of 7 and $D_{s}$ varies from 20 to $130 \mathrm{~m}$ over this time period and, thus, the dynamic range of $\left\langle w^{2}\right\rangle$ is broad. Surprisingly, simple scaling by $u_{*}$ captures much of the variation in $w$ variance. On the left side of the storm track, the maximum $w$ variance at first tracks its counterpart on the right-hand side, then gradually decays, reaches a minimum value at the time of maximum winds, and gradually recovers in the hurricane wake. In the simulations without vortex force, the maximum $w$ variance is nearly independent of the track position and is similar to what is expected for a neutral flat-wall boundary layer flow.

The differences between the variations on the right and left hand side of the storm can be traced to the wave fields, the wind-Stokes alignment shown in Figs. 5 and 6, and the production of $w$ variance by Stokes drift. The Stokes contribution to the ensemble average $w$ variance budget consists of two terms

$$
\mathcal{P}_{s} \equiv \mathcal{P}_{s}^{(u)}+\mathcal{P}_{s}^{(v)}=-\left\langle u^{\prime} w\right\rangle \frac{\partial u_{s}}{\partial z}-\left\langle v^{\prime} w\right\rangle \frac{\partial v_{s}}{\partial z},
$$

where $\left\langle u^{\prime} w\right\rangle$ and $\left\langle u^{\prime} w\right\rangle$ are ensemble average (total) vertical momentum fluxes. Note there is no mean shear production in the $w$ budget. The derivation of (10) follows the same sequence of steps used to derive the TKE 


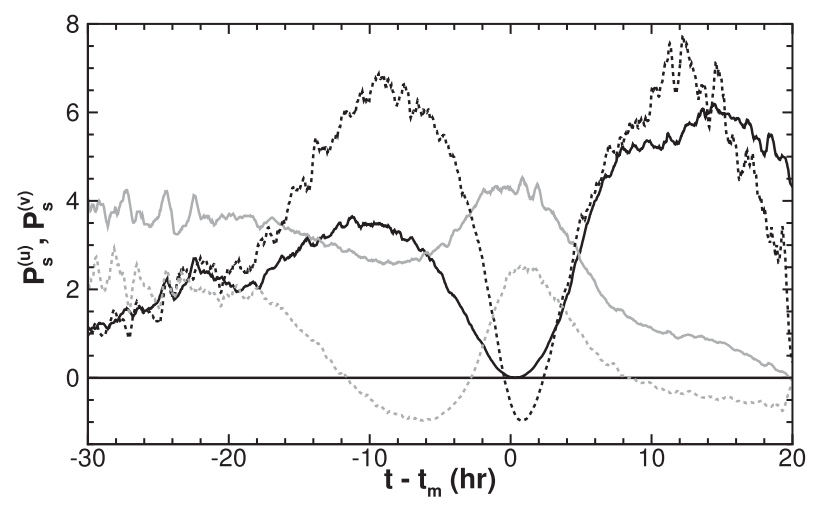

FIG. 9. Production of vertical velocity variance by Stokes drift. The terms $\mathcal{P}_{s}^{(u)}=-\left\langle u^{\prime} w\right\rangle \partial u_{s} / \partial z$ and $\mathcal{P}_{s}^{(v)}=-\left\langle v^{\prime} w\right\rangle \partial v_{s} / \partial z$ are computed at the vertical location of maximum vertical velocity variance along the right-hand side (solid lines) and left-hand side (dotted lines) of the storm track: $\mathcal{P}_{s}^{(u)}\left(\mathcal{P}_{s}^{(v)}\right)$ are indicated by black (gray) line colors. The terms are made dimensionless by $\left|h_{i}\right| / u_{*}^{3}$ and are obtained by spatial averaging over an $x-y$ plane and by time averaging over $45 \mathrm{~min}$. The momentum fluxes, which appear in $\left(P_{s}^{(u)}, P_{s}^{(v)}\right)$, are the sum of the LES resolved and SGS contributions. Note the periods where $\left(\mathcal{P}_{s}^{(u)}, \mathcal{P}_{s}^{(v)}\right)$ are negative on the left-hand side of the storm track indicating that the turbulent fluxes and gradients of Stokes drift are countergradient.

budget with wave effects (e.g., McWilliams et al. 1997). The total TKE budget depends on the vertical gradients of the currents and Stokes drift, i.e., the mean Lagrangian shear

$$
\mathcal{L}_{s}=\frac{\partial}{\partial z}\left\langle\bar{u}+u_{s}\right\rangle \hat{\mathbf{x}}+\frac{\partial}{\partial z}\left\langle\overline{\boldsymbol{v}}+v_{s}\right\rangle \hat{\mathbf{y}}
$$

Notice that in (10) the production term $\mathcal{P}_{s}$ depends on the correlation between turbulent fluxes and Stokes drift gradients. These terms can be positively correlated as in the case of wind wave equilibrium, uncorrelated or even negatively correlated: that is, the momentum fluxes can be countergradient to the Stokes drift gradient. A time series of Stokes production, given by (10), at the location of the maximum vertical velocity variance is shown in Fig. 9. There is a significant temporal correlation between the enhancement and reduction in $w$ variance, shown in Fig. 8, with the time variation of Stokes production. In particular the variance on the right and left hand sides of the storm track first begin to depart at $t-t_{m}=-12 \mathrm{~h}$. At this time on the left-hand side of the storm, the production term $\mathcal{P}_{s}^{(u)}$ reaches a positive maximum and $\mathcal{P}_{s}^{(v)}$ switches sign and becomes a destruction term. An even steeper reduction in $w$ variance occurs at the time of maximum winds where $\mathcal{P}_{s}^{(u)}$ is a sink of $w$ variance and $\mathcal{P}_{s}^{(v)}$ is small and positive. On the righthand side of the storm $\mathcal{P}_{s}^{(u, v)}$ are always positive with $\mathcal{P}_{s}^{(u)}$ reaching a maximum in the storm wake. Thus Stokes production is most often a significant source of $w$ variance, but can in certain misaligned wind wave states be a sink.
Wave-current interactions, which are key processes in the budgets of turbulence, play a role in the enhancement and reduction of $w$ variance in the hurricane OBL. The results emphasize the importance of the vertical gradients of the Stokes drift profile and their alignment with the vertical momentum fluxes. It is worth noting that $\mathcal{P}_{s}^{(u, v)}<0$ also implies that the currents and waves are moving in opposite directions near the surface (see section 6b), which can enhance wave breaking (Melville 1996).

D'Asaro (2001), using drifting Lagrangian floats, reports measurements of vertical velocity variance that are "remarkably well parameterized by wind stress" with values ranging from 1.75 to 2 times that measured in solid-wall turbulent layers driven by shear. Our vortexforce simulations on the right hand side of the storm track are in good agreement with these measurements, and we speculate that the observed high correlation with wind stress is a consequence of the tight relationship between surface Stokes drift and $u_{*}$ for approximately aligned winds and waves, as shown in Fig. 5. Harcourt and D'Asaro (2008) and Grant and Belcher (2009) propose a velocity scale for Langmuir turbulence, $w_{L}=u * / \mathrm{La}_{t}^{2 / 3}$. However our range of $\mathrm{La}_{t}$ is somewhat limited along $X=60 \mathrm{~km}$ because of the tight correlation between $\mathbf{u}_{s}$ and $u_{*}$; hence along this track $w_{L} \sim u_{*}$. We mention that the applied wind forcing in our simulations varies smoothly with time (there is no assumption of wind wave equilibrium) and then the wave fields adjust naturally to the changing local wind conditions. Hence the Stokes drift velocity imposed in the LES also varies smoothly with time and changing winds.

The impact of Langmuir turbulence on the third-order vertical velocity moment is impressive. Figure 8 depicts the minimum $\left\langle\bar{w}^{3}\right\rangle_{\min }$ based on the resolved vertical velocity. With vortex force the minimum third-order moment can be 10 times larger than its no vortex force counterpart at the time of maximum winds. Again we notice a significant reduction in $\left\langle\bar{w}^{3}\right\rangle_{\min }$ on the left-hand side of the storm in the simulation that includes the vortex force mirroring the reduction in $\left\langle w^{2}\right\rangle_{\text {max }}$. The third-order moment of vertical velocity indicates an abundance of Langmuir cells, and is important as it appears in the turbulent kinetic energy equation. The changes in $\left\langle\bar{w}^{3}\right\rangle$ indicate that Langmuir turbulence has modified the vertical transport, the divergence $\partial\left\langle\overline{w w}^{2}\right\rangle / \partial z$, in the OBL in significant ways (Grant and Belcher 2009). We find that the moments $\left\langle w^{2}\right\rangle_{\max }$ and $\left\langle\bar{w}^{3}\right\rangle_{\min }$ are stronger and clearer indicators of Langmuir circulations than the nondimensional skewness $S_{w}=\left\langle\bar{w}^{3}\right\rangle /\left\langle\bar{w}^{2}\right\rangle^{3 / 2}$, which is often used to describe Langmuir turbulence (e.g., Li and Garrett 1993; McWilliams et al. 1997; Sullivan et al. 2007). In our simulation with vortex force on the right hand side of the storm minimum skewness varies over the range 
$-1.1<S_{w}<-0.6$, which indicates that the downwelling velocities are narrower and stronger compared to the broader upward motions.

\section{b. Flow structures}

Flow visualization of the vertical velocity field provides insight into the formation and evolution of the coherent structures in a high-wind hurricane driven OBL. Extensive interrogation of the LES volumes shows that elongated streaky structures, that is, coherent Langmuir cells, are pervasive at all stages of the storm evolution. The elliptical shaped cells meander randomly in their long-axis direction with occasional merges at downstream junctions. Figure 10 is a typical sample that illustrates these processes. This image compares the instantaneous normalized vertical velocity $\bar{w} / u *$ at the time of maximum winds on the left and right hand sides of the storm. Also, included in this image are the directions of the surface and Stokes drift velocity, which provide a reference for the cell alignment. To allow a fair comparison of the spatial scales, the visualization is depicted over equal subareas of the computational domain. Notice the impressive size and strength of the cells on the right hand side of the storm. At times their long-axis is more than $100 \mathrm{~m}$ with pockets of downwelling velocity exceeding $5 u_{*} \sim 0.35 \mathrm{~m} \mathrm{~s}^{-1}$ : similar large values were observed by vertical profiling floats on the right hand side of Hurricane Frances (T. B. Sanford 2011, personal communication). Meanwhile, on the left hand side coherent cells can be identified but they are clearly weaker in magnitude and smaller in scale. This flow visualization is consistent with the temporal evolution of vertical velocity variance and third-order moment discussed previously.

Instantaneous images of $w$ illustrate the chaotic interaction between cells and turbulence. However, in order to more clearly identify the average coherent structure (a Langmuir cell) in our simulations we employ the technique of linear stochastic estimation (LSE) (e.g., Adrian et al. 1989; Adrian 1996; Christensen and Adrian 2001). LSE is a robust technique for identifying structures in turbulent flows and is an excellent approximation of a conditional average that utilizes only unconditional two-point correlation data. For our flows, we estimate the deviation from the mean horizontal currents, that is, the horizontal perturbation velocities $\mathbf{u}_{\perp}^{\prime}=\left(u^{\prime}, v^{\prime}, 0\right)$ at $\mathbf{x}^{\prime}=\mathbf{x}+\Delta \mathbf{x}$ where $\Delta \mathbf{x}$ is the spatial displacement, by the conditional average

$$
\left\langle\mathbf{u}_{\perp}^{\prime}\left(\mathbf{x}^{\prime}\right) \mid w(\mathbf{x})\right\rangle \approx \frac{\left\langle\bar{w}(\mathbf{x}) \mathbf{u}_{\perp}^{\prime}\left(\mathbf{x}^{\prime}\right)\right\rangle}{\langle\bar{w}(\mathbf{x}) \bar{w}(\mathbf{x})\rangle} \bar{w}(\mathbf{x}) .
$$

We choose resolved vertical velocity $\bar{w}(\mathbf{x})$ at the vertical location of $\left\langle w^{2}\right\rangle_{\max }$, that is, $z=z_{\max }$, as the event indicator. LSE utilizes unconditional two-point spatial correlations

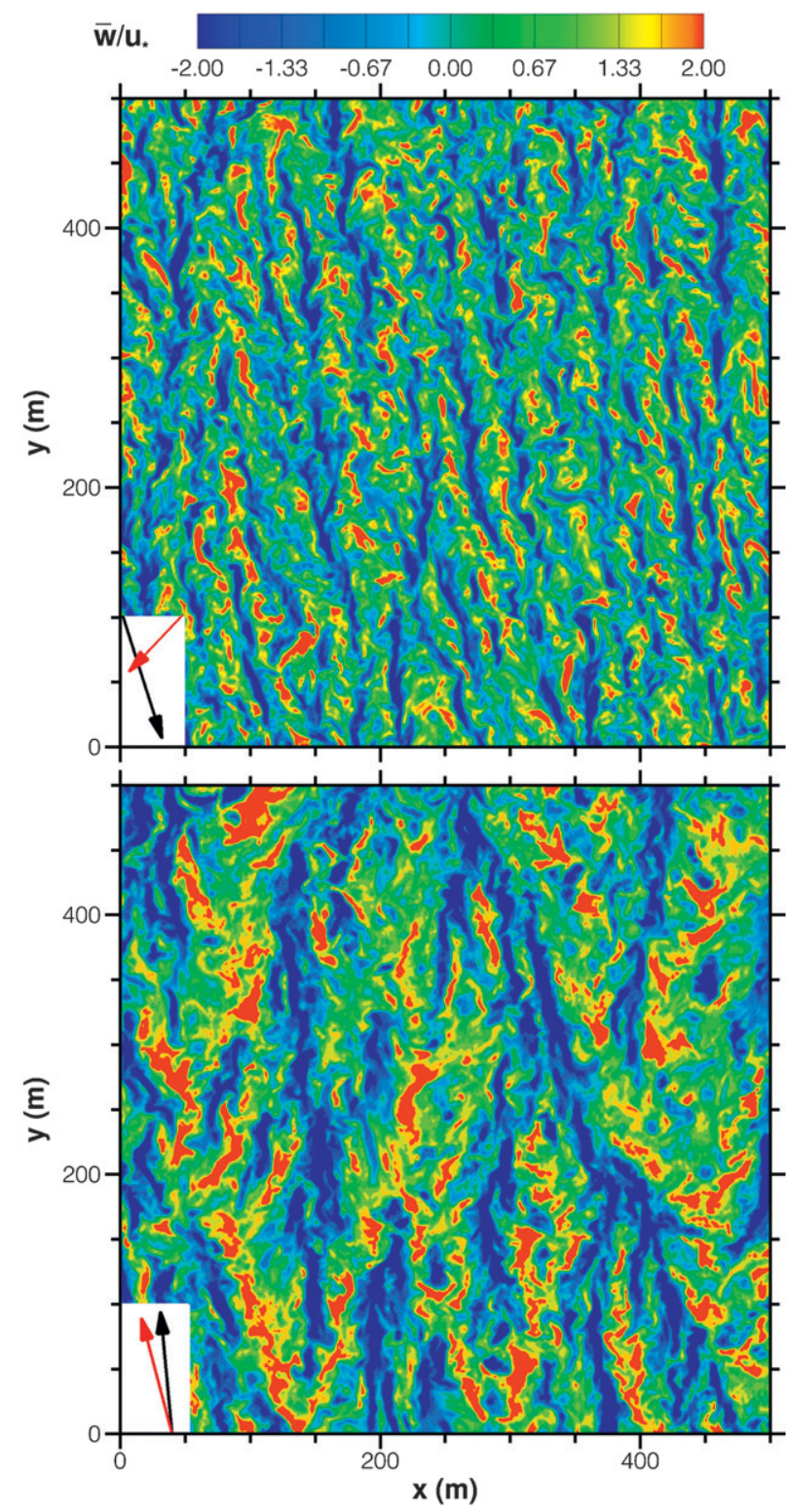

FIG. 10. Contours of vertical velocity normalized by $u_{*}$ at the time of maximum winds at (top) $z=-7.2 \mathrm{~m}$ along the track $X=-60 \mathrm{~km}$ and (bottom) at $z=-12.6 \mathrm{~m}$ along the track $X=60 \mathrm{~km}$. The subarea depicted in the top (bottom) panels is 44 (11) \% of the total $x-y$ area in their respective computational domains. The black (red) vectors are the directions of the surface wind (Stokes drift).

$$
\begin{aligned}
& R_{w \mathbf{u}_{\perp}}\left(x^{\prime}, y^{\prime}, z\right)=\frac{\left\langle\bar{w}\left(x, y, z_{\text {max }}\right) \mathbf{u}_{\perp}^{\prime}\left(x^{\prime}, y^{\prime}, z\right)\right\rangle}{\sigma_{w}\left(z_{\max }\right) \sigma_{\mathbf{u}_{\perp}}(z)} \\
& R_{w w}\left(x^{\prime}, y^{\prime}, z\right)=\frac{\left\langle\bar{w}\left(x, y, z_{\text {max }}\right) \bar{w}\left(x^{\prime}, y^{\prime}, z\right)\right\rangle}{\sigma_{w}\left(z_{\max }\right) \sigma_{w}(z)},
\end{aligned}
$$

where $\sigma$ denotes the root-mean square of the given quantity. In our implementation of (12) and (13) with 

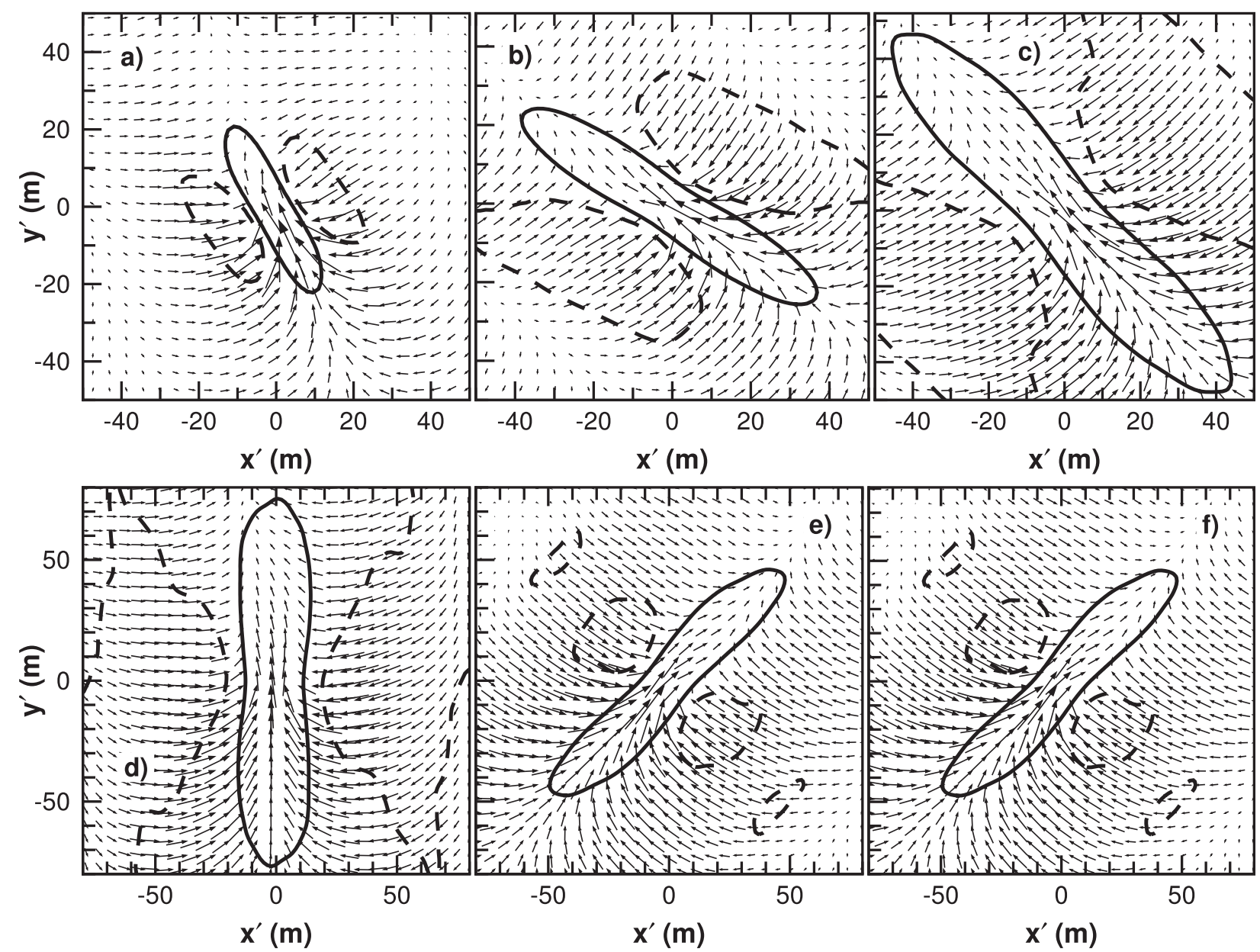

FIG. 11. Normalized two-point spatial correlation $R_{w w}\left(x^{\prime}, y^{\prime}, z_{\max }\right)$ along the track $X=60 \mathrm{~km}$. The solid (dotted) contour levels are 0.1 $(-0.05)$. The positive contour level encloses a region of strong downwelling while the negative contour level encloses a broad region of weaker upwelling. The vectors (arrows) show the magnitude and direction of the horizontal perturbation velocities $\left(u^{\prime}, v^{\prime}\right)$ induced by a Langmuir cell. (a)-(f) $t-t_{m}=(-30.2,-12.5,-3.04,-0.003,3.37,12.8) \mathrm{h}$, repectively. Note the change in the horizontal and vertical scales between the lower and upper panels.

LES data the angle brackets denote an average over an $x-y$ plane for each particular point $\left(x^{\prime}, y^{\prime}\right)$.

Time sequences of the conditionally averaged field $\mathbf{u}_{\perp}^{\prime}$ at $z=z_{\max }$ and contours of the normalized two-point spatial correlation $R_{w w}\left(x^{\prime}, y^{\prime}, z_{\max }\right)$ are provided in Figs. 11 and 12 . We define the spatial extent of the coherent Langmuir structure by contours of $R_{w w}$; the positive contour level 0.1 encompasses an ellipsoidal region of strong downwelling with side lobes of broad weaker upwelling, contour level -0.05 . Inside a downwelling region, the perturbation velocity field $\mathbf{u}_{\perp}^{\prime}$ is observed to be closely aligned (parallel) to the major axis of the elliptical shaped structure. The coherent structure defined by the two-point correlation function $R_{w w}$ and the accompanying perturbation velocity fields are a consistent average representation of the instantaneous flow fields shown previously. The flow patterns are in good agreement with the findings of McWilliams et al. (1997) and Sullivan et al. (2007), and are consistent with the CL2 instability mechanism described by Leibovich (1983). Two of the most prominent features of the cells in the hurricane OBL are their rotation and scale expansion and contraction for varying time and track position. The flow patterns exhibit strong horizontal convergence perpendicular to the major axis of the cell center. There is vigorous inflow at the cell tail and weaker outflow at the cell head with the strongest perturbation velocities centered just above the largest downwelling motions. On the left-hand side, the cells rotate continuously in counterclockwise fashion. Close inspection of the flow pattern at $t-t_{m} \sim 0$ shows the development of a vertical rotational motion that displaces the perturbation velocity vectors slightly off center. Van Roekle et al. (2012), in idealized LES with misaligned winds and waves, finds a similar result and attributes this 

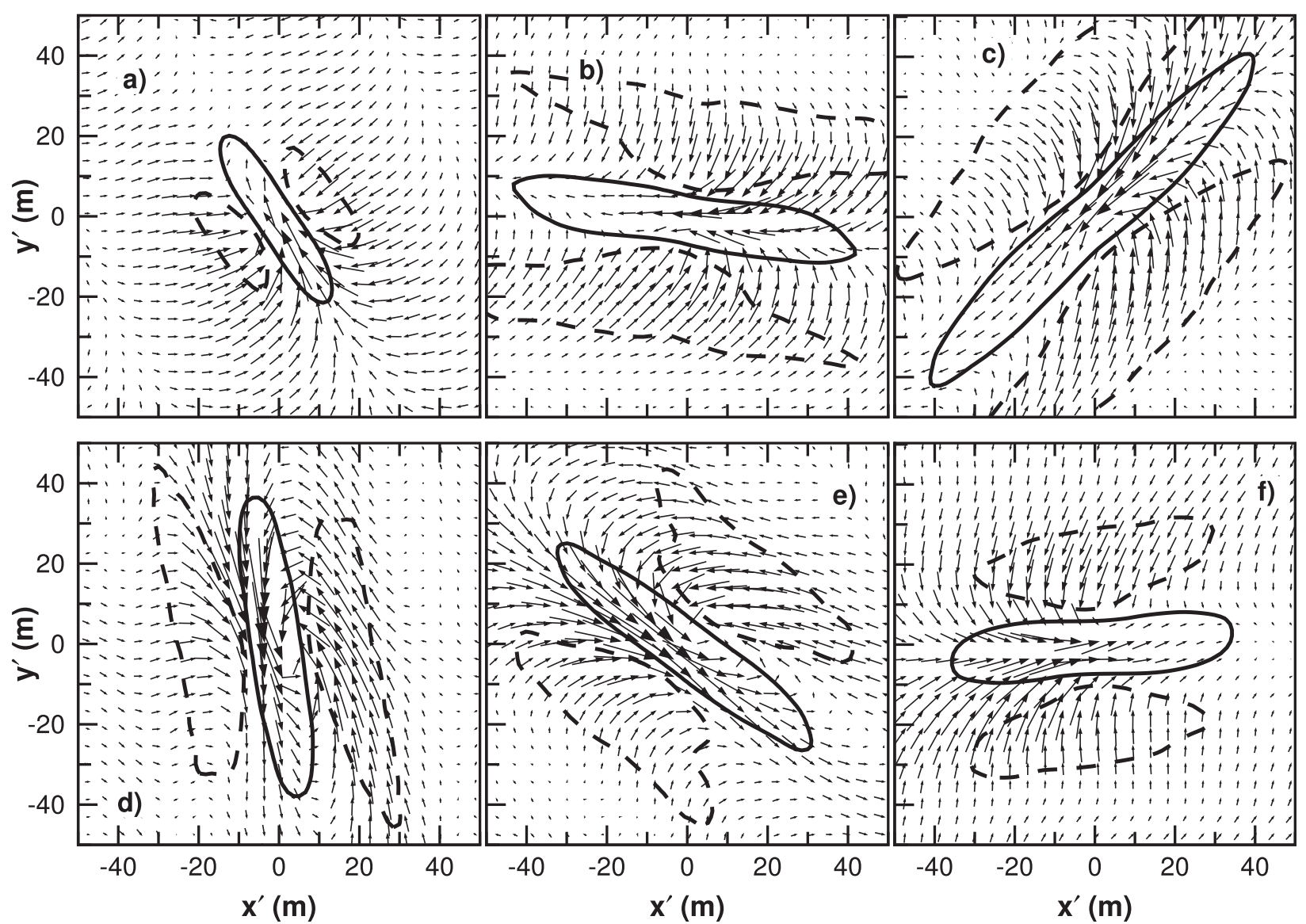

FIG. 12. The normalized two-point spatial correlation $R_{w w}\left(x^{\prime}, y^{\prime}, z_{\max }\right)$ and perturbation horizontal velocity $\left(u^{\prime}, v^{\prime}\right)$ along the track $X=-60 \mathrm{~km}$ : contour levels and labeling as in Fig. 11; (a)-(f) correspond to $t-t_{m}=(-30.9,-12.7,-3.44,-0.003,2.55,10.6) \mathrm{h}$, respectively. Note how the Langmuir cells rotate counterclockwise in contrast to those in Fig. 11.

to advection of horizontal vorticity across the cell. On the right hand side of the storm, the cells first rotate counter clockwise then rapidly clockwise. The cells grow rapidly in scale near the time of maximum winds as they tend to align themselves with the surface winds. In contrast, the cell growth is much more modest on the left hand side of the storm, consistent with the temporal changes in the vertical velocity moments. On the left hand side, the cells are larger at $t-t_{m}=(-12.7,-3.44) \mathrm{h}$ than they are at the time of maximum winds.

\section{c. Langmuir cell alignment}

One of the important questions is the orientation of a Langmuir cell for misaligned winds and waves. Cell orientation significantly complicates the development of OBL parameterizations with wave effects (McWilliams et al. 2012). In Fig. 13, we show the surface wind and Stokes drift velocities and the direction of the Lagrangian shear given by (11). The orientation of the cells in LES is deduced from the conditionally averaged velocity fields sampled at the cell center $\left[u^{\prime}\left(0,0, z_{\max }\right), v^{\prime}\left(0,0, z_{\max }\right)\right]$. The Langmuir shear $\mathcal{L}_{s}$ is a key quantity in Langmuir turbulence as it appears as a production term in the turbulent kinetic energy equation [see Eq. (5.1) from McWilliams et al. (1997) and Grant and Belcher (2009)]. In addition, Holm (1996) shows how the Lagrangian shear contributes to vortex stretching, that is, the tilting of vertical vorticity into streamwise directions. The results in Fig. 13 indicate that the cells are oriented primarily with $\mathcal{L}_{s}$, which is also broadly parallel to the surface wind direction. The deviation from $\mathcal{L}_{S}$ is largest along $X=60 \mathrm{~km}$ in the wake where the cell alignment tends more toward the wind direction. Figure 14 shows how $\mathcal{L}_{s}$ evolves with time at the water surface. Notice that there are periods when Stokes and current shear are of opposite sign, which leads to a reduction in turbulence variances (see discussion in section 5a).

\section{Impacts of Langmuir turbulence on OBL statistics}

In the hurricane driven OBL, coherent Langmuir cells interacting with background turbulence leave an imprint on 

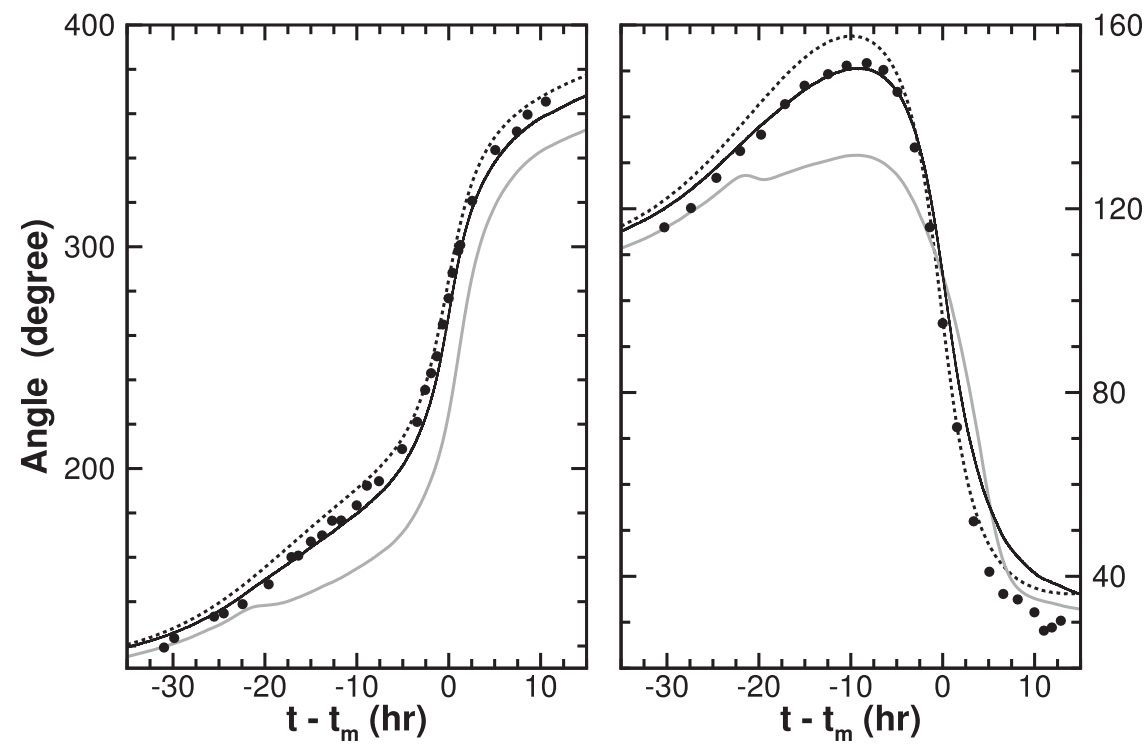

FIG. 13. Time variation of the surface wind (dotted black line), the surface Stokes drift velocity (gray line), and the Lagrangian shear (black line): (left) $X=-60 \mathrm{~km}$ and (right) $X=$ $60 \mathrm{~km}$. The dominant Langmuir cell direction deduced from the LES are indicated by solid circles. Angles are measured counterclockwise from the $x$ axis.

the vertical velocity. Langmuir turbulence also effects the OBL low-order statistics, namely, mean currents, temperature profiles, momentum and heat fluxes, variances and dissipation. This is an overly abundant amount of information to present, and thus we focus on a few select statistics that demonstrate the role of wave-current interactions.

\section{a. Horizontal variances}

In simulations with steady aligned winds and waves the coherent Langmuir cells modulate the horizontal streamwise and spanwise variances compared to their flat-wall counterparts. The streamwise oriented cells induce strong spanwise convergence at downwelling sites and their vortical rotation about the $x$ axis amplifies the spanwise variance $\left\langle\boldsymbol{v}^{\prime 2}\right\rangle$ at the expense of streamwise variance $\left\langle u^{\prime 2}\right\rangle$. For example, McWilliams et al. (1997) reports that, based on the resolved LES velocities, the ratio $\left\langle\overline{\boldsymbol{v}}^{\prime 2}\right\rangle /\left\langle\bar{u}^{\prime 2}\right\rangle>3$ near the water surface; theoretical linearized rapid distortion results for a propagating surface wave are in qualitative agreement with this numerical result (Teixeira and Belcher 2002). Wave breaking, however, tends to weaken this variance anisotropy by disrupting the near-surface cell structure (Noh et al. 2004; Sullivan et al. 2007). This intercomponent exchange of horizontal variance is also present in our simulations and reflects the directional changes in the time varying winds and waves as depicted in Fig. 15. As shown previously, cells tend to align with the time dependent Lagrangian shear direction and the rotating cells induce a smooth exchange of variance between the horizontal velocity components. The cells also noticeably alter the phase relationship between the resolved horizontal variances so that $\left\langle\bar{u}^{\prime 2}\right\rangle$ and $\left\langle\bar{v}^{\prime 2}\right\rangle$ reach maximum values at times markedly different than simulations without vortex force. This is especially apparent at the time of maximum winds on the right hand side of the storm track. In the absence of vortex force $\left\langle\bar{u}^{\prime 2}\right\rangle$ is a minimum at $t-t_{m}=0$, while it reaches a maximum when vortex force is included. Note that at $t-t_{m}=0$ the cells are primarily oriented in the

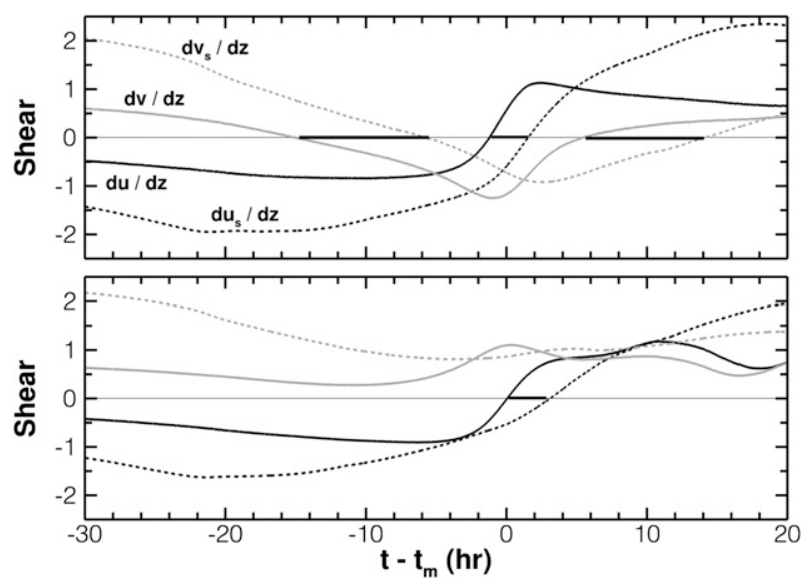

FIG. 14. Mean Lagrangian shear components at the water surface on (top) the left-hand side and (bottom) right-hand side of the storm. The shears are made dimensionless by $z_{1} / u_{*}$, where $z_{1}$ is the location of the first LES gridpoint below the water surface; the heavy black lines indicate time periods where the current and Stokes shears have opposite signs. 


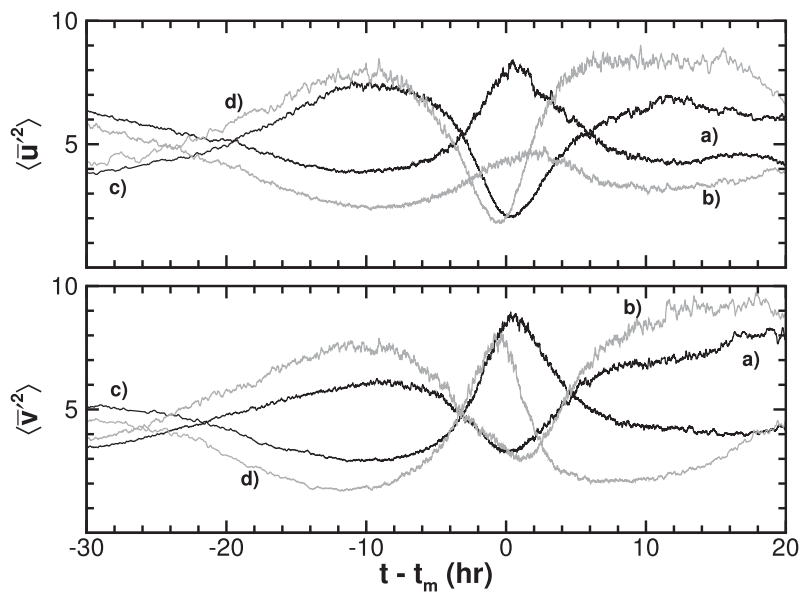

FIG. 15. Temporal variation of the resolved horizontal variances $\left\langle\bar{u}^{\prime 2}, \bar{v}^{\prime 2}\right\rangle$ at $z=-0.5 \mathrm{~m}$ below the water surface for simulations with and without wave effects at locations left and right of the storm center: simulations with wave effects for (a) $X=60 \mathrm{~km}$ and (b) $X=-60 \mathrm{~km}$ and with no wave effects for (c) $X=60 \mathrm{~km}$ and (d) $X=-60 \mathrm{~km}$. As the winds and waves veer with time the horizontal component with maximum variance also shifts tracking the rotation of the coherent Langmuir cells. At $t-t_{m}=0$ the winds are primarily aligned with the $y$ direction and then the $v$ component is a maximum as might be expected for a wall-bounded shear flow without wave effects. The inclusion of vortex force alters this relationship so that the $u$ component is a maximum.

$y$ direction, which results in an amplification of the $u$ variance with a reduction in the $v$ variance. Our flow visualization shows that the cells are weaker and smaller in scale on the left-hand side of the storm track and this results in a lower value of $u$ variance compared to its counterpart on the right-hand side near the water surface.

\section{b. Current and variance vertical profiles}

Vertical profiles of the mean currents $\langle\bar{u}, \bar{v}\rangle$ highlight the complex coupling that occurs between winds and waves and between waves and currents. Figure 16 compares the mean currents and Stokes drift velocity, normalized by local $u_{*}$, on the left and right sides of the storm track for simulations at the time of maximum winds. Wave influences are pronounced. On the right-hand side the Stokes drift velocity is dominated by the $v_{s}$ component with the wind-Stokes alignment near unity over a sizeable fraction of the OBL (see lower panel of Fig. 5). The potent Langmuir cells induce a vertically uniform $v$-current profile down to a depth $z /|h| \sim 0$.6. In contrast, in the simulation without wave effects the vertical gradient $\partial\langle\bar{v}\rangle / \partial z$ is surface intensified but varies continuously over the entire OBL. In cases with and without wave effects, there are steep current gradients near the base of the OBL.

Inspection of the current profiles shows that Stokes drift induces a return Eulerian flow opposite to the direction of wave propagation: this is a consequence of Stokes-Coriolis coupling, $f \hat{\mathbf{z}} \times \mathbf{u}_{s}$, in the LES equations (Huang 1979; Gnanadesikan and Weller 1995; McWilliams et al. 1997; Polton et al. 2005). On the right-hand side of the storm this process leads to Eulerian current changes that are similar to simulations with aligned winds and waves. However, on the left-hand side of the storm with wind-wave misalignment, the wave-induced return flow leads to oppositely signed gradients $\partial\langle\bar{u}\rangle / \partial z$ and $\partial u_{s} / \partial z$. As discussed in section 5a, the vertical momentum flux $\left\langle u^{\prime} w\right\rangle$ is then countergradient to the gradient of Stokes drift, which inhibits the production of $w$ variance. Over the bulk of the OBL the currents with and without wave effects are broadly similar with the important changes occurring near the water surface.

The resolved velocity variance profiles displayed in Fig. 17 paint a consistent picture of the impact of Langmuir turbulence in the nonequilibrium hurricane regime. On the left-hand side of the storm at $t-t_{m}=0$, the $w$ variance in the simulation with vortex force displays a slightly elevated subsurface maximum compared to its counterpart without vortex force. The wave effects on the horizontal variances are confined to a shallow layer near the surface $-0.15<z /|h|<0$, and outside this region the profiles from the simulations with and without vortex force are nearly indistinguishable. This is consistent with the flow visualization and the interpretation of weak Langmuir turbulence on the left hand side of the storm discussed in section 5 . The variance profiles along $X=60 \mathrm{~km}$ are in sharp contrast to their counterparts along $X=-60 \mathrm{~km}$. The wave effects are pronounced and the changes in the $v$ and $w$ variances extend over a large fraction of the OBL, more than $50 \%$. Also, it is apparent that vertical transport by the Langmuir cells contributes to the elevated $v$ variance in the region $-1<z /|h|<-0.5$. This hints at a coupling with shear instabilities at the thermocline induced by inertial resonance.

\section{c. Entrainment flux and SST}

One of the primary bulk functions of the hurricane OBL is to entrain cool water from below the thermocline into the mixed layer. Over time, this lowers SST that can, depending on the translation speed of the storm, feedback onto the large-scale hurricane dynamics. Previous modeling and observational studies (e.g., Price 1981; Crawford and Large 1996; Skyllingstad et al. 2000; Sanford et al. 2011) find that the primary dynamical mechanism for entraining cool water is shear instability at the base of the OBL induced by inertially rotating wind stress (see the upper panel of Fig. 5). This critical dynamical process is also found to be dominant in our simulations but is augmented by additional mixing induced by the coherent Langmuir cells that develop 

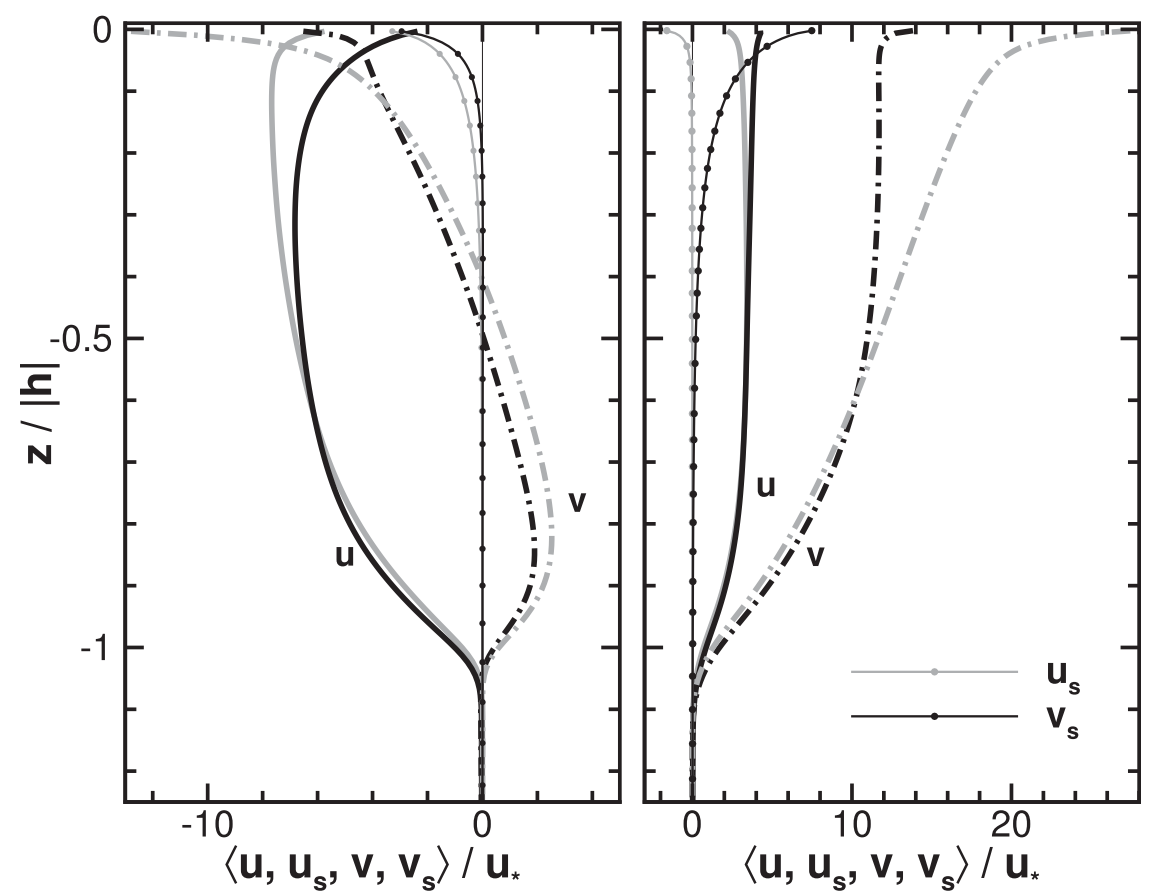

FIG. 16. Profiles of mean currents $\langle\bar{u}, \bar{v}\rangle$ and Stokes drift velocity $\left\langle u_{s}, v_{s}\right\rangle$ normalized by $u_{*}$ at the time of maximum winds: left (right) sides of the storm are shown in left (right) panels. The black (gray) colors are simulations with (without) vortex force. Averages are computed over an $x-y$ plane and $3600 \mathrm{~s}$ in time.

in the OBL on both sides of the storm track. This is illustrated in Fig. 18 where we compare the minimum total heat flux at the base of the OBL, that is, the entrainment flux $\left\langle w \theta^{\prime}\right\rangle_{\min }$, and the change in SST, $\Delta \theta=\langle\theta\rangle-\theta_{i}$ at $z=0$, for simulations with and without wave effects. The entrainment flux $\left\langle w \theta^{\prime}\right\rangle_{\min }$ is the sum of LES resolved and SGS contributions and is simply computed by sweeping through the vertical profile of the mean total temperature (or heat) flux and locating its minimum value. Vertical profiles of mean temperature and heat flux are obtained by averaging over an $x-y$ plane in the LES domain and $1000 \mathrm{~s}$ in time.

Far ahead of the storm center the normalized entrainment flux $\left\langle w \theta^{\prime}\right\rangle_{\min } / Q_{*}(X, t) \sim-0.25$, which is slightly more negative than the classical value of -0.2 for a daytime convective atmospheric boundary layer (e.g., Schmidt and Schumann 1989; Moeng and Sullivan 1994). This is expected since the Stokes drift is modest and the OBL is in a regime of mixed shear and convective forcing with the ratio $\left|h_{i}\right| / L \sim-1$. With increasing time, the OBL becomes increasingly dominated by shear and wave forcing. At the time of maximum winds, the entrainment flux exceeds its surface value by a factor of $(-10$, -3 ) on the (right, left) sides of the storm, respectively. Langmuir turbulence noticeably enhances the entrainment flux compared to the situation without wave effects. For example, on the right-hand side at $t-t_{m}=$ $-4 \mathrm{~h}$ the normalized entrainment flux increases from -4 to -6 , a $50 \%$ increase when wave effects are included. At $t-t_{m}=0$, the increase is about $20 \%$ and the resulting maximum change in $\Delta \theta$ is about $0.25 \mathrm{~K}$ cooler. In absolute values, the changes in entrainment flux and SST on the left hand side of the storm are more modest than their counterparts on the right hand side. On both sides of the storm track wave effects cause the minimum entrainment flux to occur earlier in time, perhaps by as much as $3 \mathrm{~h}$. This suggests enhanced coupling with the inertial currents at the thermocline. Our estimate that the normalized entrainment (cooling) flux on the righthand side of the storm is greater than 10 times the surface heat flux is in good agreement with the observations by Sanford et al. (2011, see p. 1044). The LES predicted changes in SST on the left and right hand sides of the storm are in good agreement with the observations of D'Asaro et al. (2007), but those observations do not readily lend themselves to quantifying the importance of surface waves.

In the hurricane wake the entrainment flux, especially on the right-hand side of the storm, appears to retreat toward its prestorm value in a series of steep rises and declines that is noticeably different than in the time period $t-t_{m}<0$. Flow visualization reveals packets of 

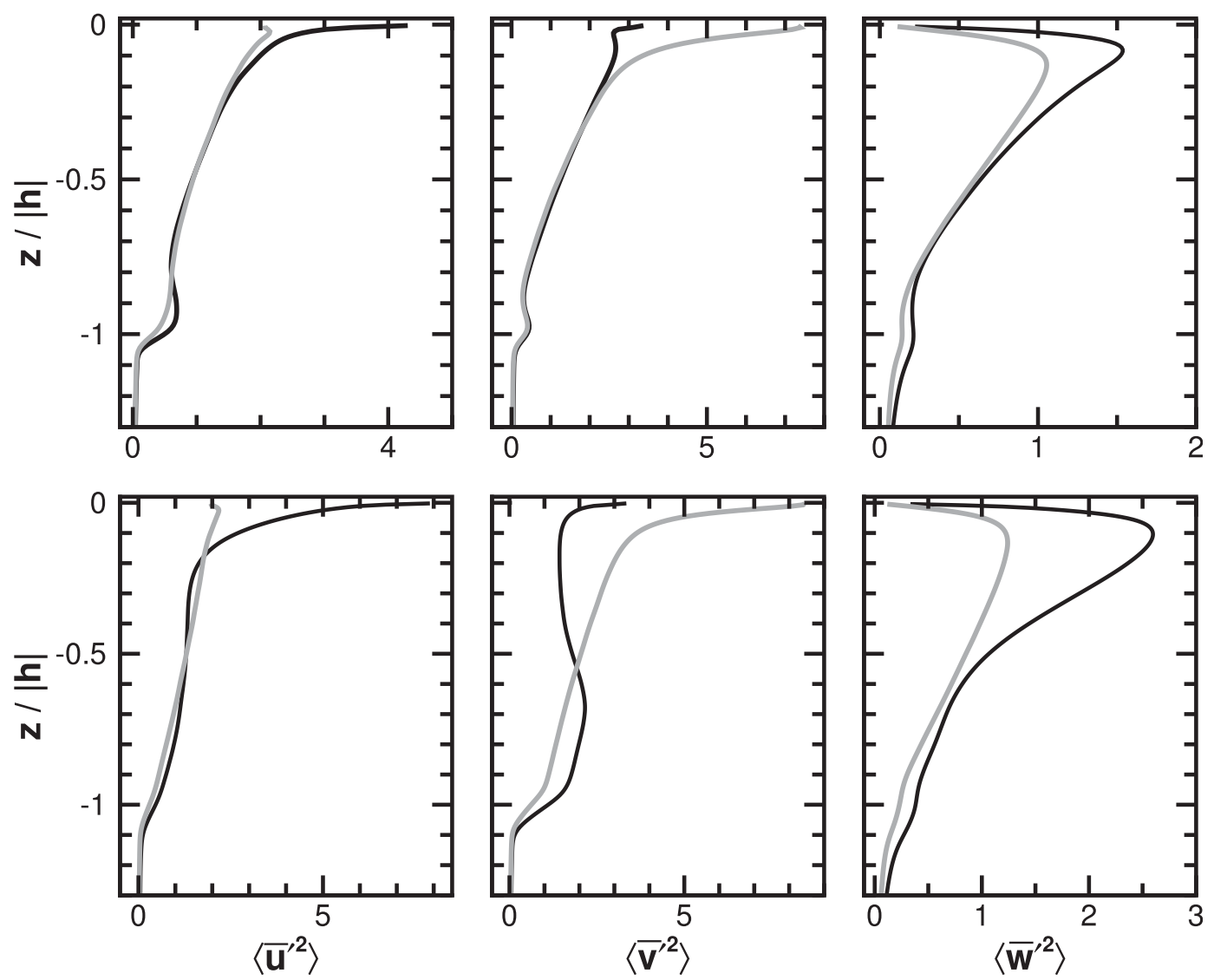

FIG. 17. (left to right) Vertical profiles of resolved variances $\left\langle\bar{u}^{\prime 2}, \bar{v}^{\prime 2}, \bar{w}^{\prime 2}\right\rangle$ normalized by $u_{*}^{2}$ for (top) left side and (bottom) right side of the storm track. The black (gray) solid lines in each figure are simulations with (without) vortex force. Results are at $t-t_{m}=0$.

large-scale propagating gravity waves that overturn intermittently, creating large oscillations in the entrainment flux as well as other OBL statistics. These turbulent gravity waves appear to be a product of the vigorous resonant wind stress forcing. The large horizontal scale of these structures motivated our use of the wide LES horizontal domain described in section $3 \mathrm{~b}$.

\section{d. Boundary layer depth and Stokes scale}

It is interesting to notice that the augmentation of the bulk temperature entrainment and SST change induced by Langmuir turbulence occurs during the time period $t-t_{m}<0$. This can be interpreted in terms of the surface $\mathrm{La}_{t}$ and the variations of the bulk OBL length scales $h$ and $D_{s}$. Figure 19 depicts the time growth of the OBL and the ratio of the Stokes depth scale to the local OBL depth, that is, the ratio $D_{s} /|h|$, along the tracks $X=$ $\pm 60 \mathrm{~km}$. The OBL depth $h$ is estimated using the maximum temperature gradient method described in Sullivan et al. (1998); essentially $h$ is the spatial average of the boundary layer depths $h(x, y)$ deduced along every vertical column in the LES grid box. A comparison of Fig. 18 with Fig. 19 shows that the largest impact of Langmuir turbulence on the entrainment flux occurs in the deepening phase of the OBL, that is, when the ratio $D_{s} /|h|>0.75$; a similar result is reported by Skyllingstad et al. (2000). Based on the definition of $D_{s}$ given by (7), this empirical ratio corresponds to a nondimensional $e$ folding length scale for a monochromatic Stokes wave of $z_{L} /|h|=(3 / 16 \pi)$ or about $6 \%$ of the OBL depth. We note that in the simulations of McWilliams et al. (1997) and Sullivan et al. (2007) $D_{s} /|h| \sim 1.82$ and they find significant wave effects on entrainment. In the present simulations when $D_{s} /|h|>0.75$, the coherent cells are of sufficient strength to effectively transport momentum and kinetic energy to the base of the thermocline and thereby increase the entrainment of cool water. In the hurricane wake, $t-t_{m}>0$, the ratio $D_{s} /|h|<0.5$ because the OBL mixes to very deep levels by inertial resonance and $D_{s}$ is reduced by the falling surface winds. The ratio $D_{s}|| h \mid$ appears to be an empirical indicator of when Langmuir turbulence impacts entrainment in a stratified OBL. 


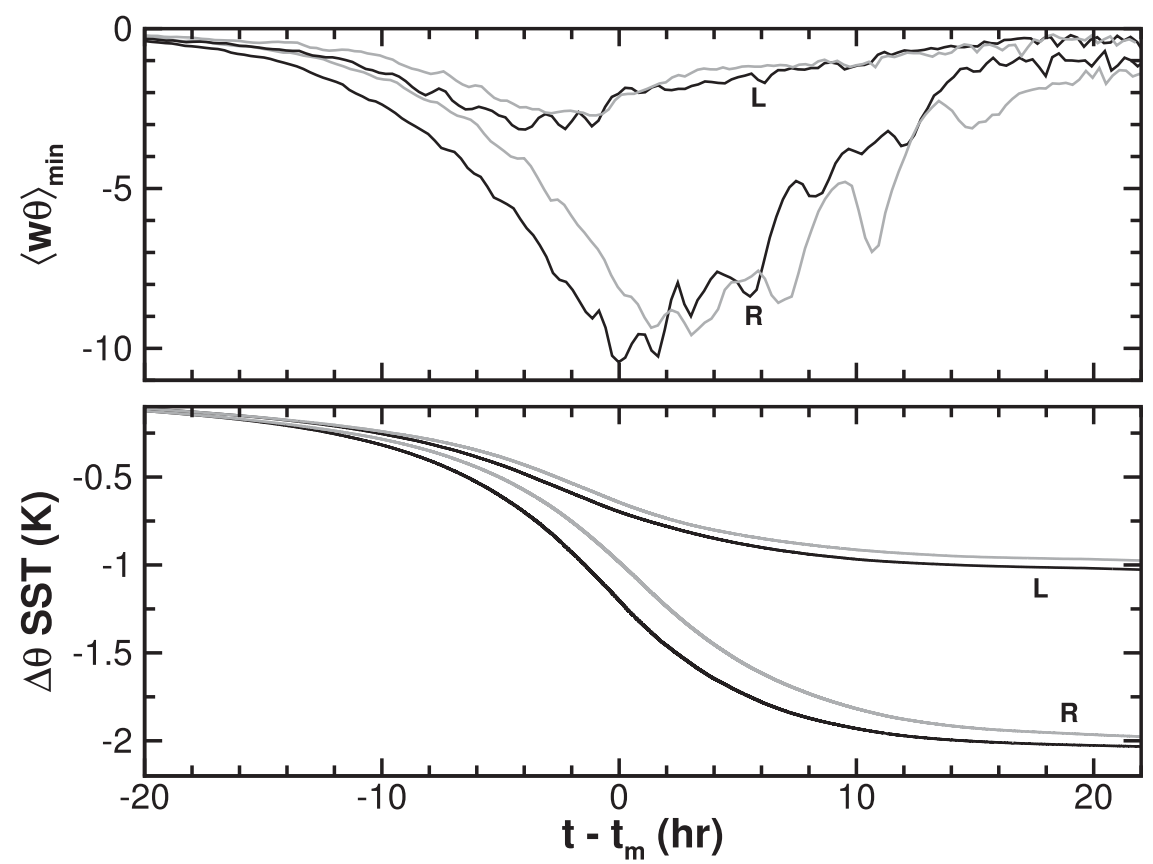

FIG. 18. Temporal variation of (top) entrainment flux and (bottom) SST change. The groups of curves marked "L" ("R") are conditions on the left (right) hand side of the storm. In each figure the black (gray) lines are simulations with (without) vortex force. The entrainment flux is normalized by $Q_{*}(X, t)$ the local value of the surface heat flux (see Fig. 4) and $\Delta \theta=\langle\theta\rangle-\theta_{i}$ at $z=0$.

\section{Summary and conclusions}

A turbulence resolving large-eddy simulation (LES) model is used to study ocean boundary layers (OBLs) driven by extreme (hurricane) winds and waves. The LES model adopts wave-averaged equations that include the vortex force $\mathbf{u}_{s} \times \overline{\boldsymbol{\omega}}$, where $\mathbf{u}_{s}$ is the wave field Stokes drift velocity and $\overline{\boldsymbol{\omega}}$ is the vorticity. Large-scale time-varying wind and wave fields input to the LES are generated by WaveWatch III; the wind and wave fields are an idealization of Hurricane Frances traveling at a speed of $5.5 \mathrm{~m} \mathrm{~s}^{-1}$. The surface wind and wave fields are in a nonequilibrium state and, as a result, the Stokes drift velocity is a complex two-component vector that varies with time, storm track position, and spirals with depth. Highresolution LES using as many as $1024 \times 1024 \times 256$ grid points integrated for more than 70 physical hours are conducted at fixed positions right and left $(X= \pm 60 \mathrm{~km})$ of the storm track.

The surface-based turbulent Langmuir number $\mathrm{La}_{t}$, which is used to assess the relative importance of shear and wave forcing, varies from 0.2 to more than 0.6 but over wide regions $\mathrm{La}_{t} \sim 0.3$. Thus the hurricane forced OBL is often in a Langmuir turbulence regime. The LES solutions exhibit important differences from previous simulations that adopt wind wave equilibrium. The potency of Langmuir turbulence and the size and strength of the coherent Langmuir cells depends on the locally evolving wind wave state. At the time of maximum winds on the right-hand side of the storm, the near inertial rotation of the winds creates a "resonant" Stokes drift that is well aligned with the surface winds over a large fraction of the OBL. Imagery shows that the resulting instantaneous Langmuir cells can be more than $100 \mathrm{~m}$ in

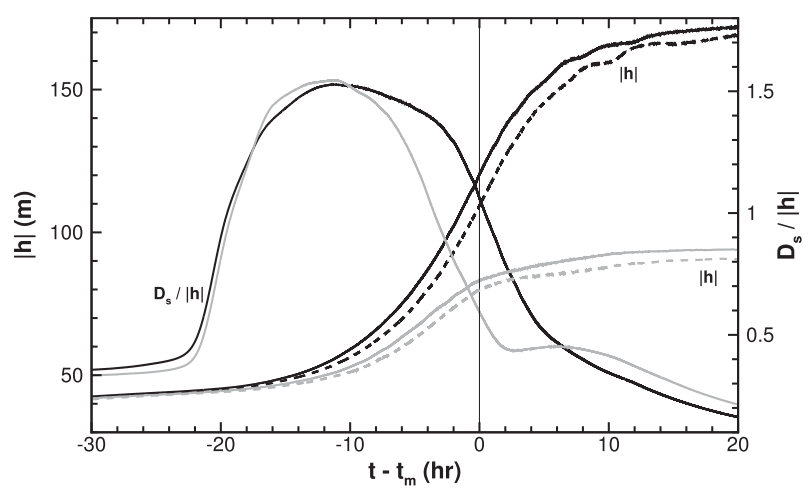

FIG. 19. Temporal variation of the OBL depth $|h|$ (left ordinate) and ratio $D_{s} /|h|$ (right ordinate). The solid black (gray) lines are simulations with vortex force on the right (left) side of the storm. The Stokes depth scale $D_{s}$ is predicted by (7). The dashed black (gray) lines are estimates of $|h|$ on the right (left) side for simulations with no vortex force. For reference, the LES by McWilliams et al. (1997) is carried out with the ratio $D_{s} /|h| \sim 1.82$. 
length with downwelling velocities exceeding $5 u_{*}\left(u_{*}\right.$ is the friction velocity) and are depth filling. The cells are much weaker and smaller on the left-hand side because of wind wave misalignment. The latter results in oppositely signed vertical gradients of current and Stokes drift. Hence the vertical momentum fluxes can be countergradient to $\partial \mathbf{u}_{s} / \partial z$, which results in less production of vertical velocity variance near the water surface. Vertical momentum fluxes countergradient to vertical gradients of Stokes drift are one of many possible regimes in nonequilibrium winds and waves. The average coherent Langmuir cell, identified using linear stochastic estimation, rotates, grows, and shrinks depending on $\mathrm{La}_{t}$, and the alignment between the surface wind and depth-varying Stokes profile. Over time on both sides of the storm track, the cells tend to closely align with the mean Lagrangian shear direction.

The vertical profiles of mean currents and temperature, turbulence variances, and momentum and heat fluxes, are impacted by Langmuir turbulence to varying degrees. At the time of maximum winds, Langmuir turbulence enhances the minimum heat flux (or entrainment flux) at the base of the OBL by $20 \%$ and lowers the sea surface temperature by $0.25 \mathrm{~K}$ on the right-hand side of the storm. Inertially rotating wind stress, however, remains the dominant dynamical process for entraining cold water into the OBL. Wave effects also produce changes to entrainment and SST on the left-hand side of the storm, but to a lesser degree. In the nonequilibrium wind wave regime studied here the surface based turbulent Langmuir number $\mathrm{La}_{t}$ appears to be insufficient in describing when wave effects impact the OBL. The shape of the Stokes drift vertical profile, wind wave alignment, and the depth scale of the Stokes profile $D_{s}$ are important bulk parameters. When the ratio $D_{s} /|h|>0.75$, where $h$ is the OBL depth, Langmuir turbulence enhances entrainment at the thermocline. Wave breaking, which is a dominant feature of the high wind OBL, is omitted here but will be considered in future work.

Acknowledgments. We thank the anonymous reviewers for their comments and suggestions that improved this work. PPS and JCM were supported by the Physical Oceanography Program through the Office of Naval Research (ONR) Departmental Research Initiatives, Impact of Typhoons on the Ocean in the Pacific (ITOP) and High Resolution Air-Sea Interaction (HiRes). PPS is also supported by the National Science Foundation through the National Center for Atmospheric Research. LR and WKM were supported by grants to WKM from NSF (Physical Oceanography) and ONR HiRes. This research used computer resources provided by NCAR and the Department of Defense High Performance Computing Modernization Program.

\section{REFERENCES}

Adrian, R. J., 1996: Stochastic estimation of the structure of turbulent fields. Eddy Structure Identification, J. P. Bonnet, Ed., Springer Verlag, 145-196.

_ B. B. Jones, M. K. Chung, Y. Hassan, C. K. Nithianandan, and A. T.-C. Tung, 1989: Approximation of turbulent conditional averages by stochastic estimation. Phys. Fluids, 1A, 992998.

Banner, M. L., I. Jones, and J. C. Trinder, 1989: Wavenumber spectra of short gravity waves. J. Fluid Mech., 25, 321-344.

Belcher, S. E., and Coauthors, 2012: A global perspective on Langmuir turbulence in the ocean surface boundary layer. Geophys. Res. Lett., doi:10.1029/2012GL052932, in press.

Bell, M. M., M. T. Montgomery, and K. A. Emanuel, 2012: Air-sea enthalpy and momentum exchange at major hurricane wind speeds observed during CBLAST. J. Atmos. Sci., 69, 3197-3222.

Black, P., and Coauthors, 2007: Air-sea exchange in hurricanes: Synthesis of observations from the Coupled Boundary Layers Air-Sea Transfer experiment. Bull. Amer. Meteor. Soc., 88, 357-374.

Capet, X., J. C. McWilliams, M. J. Molemaker, and A. Shchepetkin, 2008: Mesoscale to submesoscale transition in the California Current system. Part I: Flow structure, eddy flux, and observational tests. J. Phys. Oceanogr., 38, 29-43.

Chini, G. P., K. Julien, and E. Knobloch, 2009: An asympotically reduced model of turbulent Langmuir circulation. Geophys. Astrophys. Fluid Dyn., 103, 179-197.

Christensen, K. T., and R. J. Adrian, 2001: Statistical evidence of hairpin vortex packets in wall turbulence. J. Fluid Mech., 431, 433-443.

Craik, A., and S. Leibovich, 1976: A rational model for Langmuir circulations. J. Fluid Mech., 73, 401-426.

Crawford, G. B., and W. G. Large, 1996: A numerical investigation of resonant inertial response of the ocean to wind forcing. J. Phys. Oceanogr., 26, 873-891.

D'Asaro, E., 2001: Turbulent vertical kinetic energy in the ocean mixed layer. J. Phys. Oceanogr., 31, 3530-3537.

- 2003: The ocean boundary layer below Hurricane Dennis. J. Phys. Oceanogr., 33, 561-579.

_ T. B. Sanford, P. P. Niiler, and E. J. Terrill, 2007: Cold wake of Hurricane Frances. Geophys. Res. Lett., 34, L13604, doi:10.1029/2007GL030160.

Deardorff, J. W., 1972: Numerical investigation of neutral and unstable planetary boundary layers. J. Atmos. Sci., 29, 91-115.

Donelan, M. A., B. K. Haus, N. Reul, W. J. Plant, M. Stiassnie, H. C. Graber, O. B. Brown, and E. S. Saltzman, 2004: On the limiting aerodynamic roughness of the ocean in very strong winds. Geophys. Res. Lett., 31, L18306, doi:10.1029/ 2004GL019460.

Fan, Y., I. Ginis, T. Hara, C. W. Wright, and E. J. Walsh, 2009: Numerical simulations and observations of surface wave fields under an extreme tropical cyclone. J. Phys. Oceanogr., 39, 2097-2116.

French, J. R., W. M. Drennan, J. A. Zhang, and P. G. Black, 2007: Turbulent fluxes in the hurricane boundary layer. Part I: Momentum flux. J. Atmos. Sci., 64, 1089-1102.

Gnanadesikan, A., and R. A. Weller, 1995: Structure and instability of the Ekman spiral in the presence of surface gravity waves. J. Phys. Oceanogr., 25, 3148-3171. 
Grant, A. L. M., and S. E. Belcher, 2009: Characteristics of Langmuir turbulence in the ocean mixed layer. J. Phys. Oceanogr., 39, 1871-1887.

— , and - 2011: Wind-driven mixing below the oceanic mixed layer. J. Phys. Oceanogr., 41, 1556-1575.

Harcourt, R. R., and E. A. D'Asaro, 2008: Large-eddy simulation of Langmuir turbulence in pure wind seas. J. Phys. Oceanogr., 38, 1542-1562.

Holm, D. D., 1996: The ideal Craik-Leibovich equations. Physica $D, \mathbf{9 8}, 415-441$.

Huang, N. E., 1979: On surface drift currents in the ocean. J. Fluid Mech., 91, 119-208.

Jacob, S. D., and L. K. Shay, 2003: The role of oceanic mesoscale features on the tropical cyclone-induced mixed layer response: A case study. J. Phys. Oceanogr., 33, 649676.

,-- , A. J. Mariano, and P. G. Black, 2000: The 3D oceanic mixed layer response to Hurricane Gilbert. J. Phys. Oceanogr., 30, 1407-1429.

Kenyon, K. E., 1969: Stokes drift for random gravity waves. J. Geophys. Res., 74, 6991-6994.

Kukulka, T., A. J. Plueddemann, J. H. Trowbridge, and P. P. Sullivan, 2010: Rapid mixed layer deepening by the combination of Langmuir and shear instabilities: A case study. J. Phys. Oceanogr., 40, 2381-2400.

Large, W. G., and S. Pond, 1981: Open ocean flux measurements in moderate to strong winds. J. Phys. Oceanogr., 11, 324 336.

_ J. C. McWilliams, and S. C. Doney, 1994: Oceanic vertical mixing: A review and a model with a nonlocal boundary layer parameterization. Rev. Geophys., 32, 363-403.

Leibovich, S., 1983: The form and dynamics of Langmuir circulations. Annu. Rev. Fluid Mech., 15, 391-427.

Li, M., and C. Garrett, 1993: Cell merging and the jet/downwelling ratio in Langmuir circulations. J. Mar. Res., 51, 737-769.

$\_,-$, and E. Skyllingstad, 2005: A regime diagram for classifying turbulent large eddies in the upper ocean. Deep-Sea Res. I, 52, 259-278.

McWilliams, J. C., and J. M. Restrepo, 1999: The wave-driven ocean circulation. J. Phys. Oceanogr., 29, 2523-2540.

_ P. P. Sullivan, and C.-H. Moeng, 1997: Langmuir turbulence in the ocean. J. Fluid Mech., 334, 1-30.

_ J. R. Restrepo, and E. M. Lane, 2004: An asymptotic theory for the interaction of waves and currents in shallow coastal water. J. Fluid Mech., 511, 135-178.

_ - E. Huckle, J.-H. Liang, and P. P. Sullivan, 2012: The wavy Ekman layer: Langmuir circulations, breaking waves, and Reynolds stress. J. Phys. Oceanogr., 42, 1793-1816.

Melville, W. K., 1996: The role of wave breaking in air-sea interaction. Annu. Rev. Fluid Mech., 28, 279-321.

— L. Romero, and J. M. Kleiss, 2005: Extreme wave events in the Gulf of Tehuantepec. Rogue Waves: Proc. 14th 'Aha Huliko'a Hawaiian Winter Workshop, Honolulu, HI, University of Hawaii at Manoa, 23-28.

Moeng, C.-H., 1984: A large-eddy simulation model for the study of planetary boundary-layer turbulence. J. Atmos. Sci., 41, 2052 2062.

_- and P. P. Sullivan, 1994: A comparison of shear and buoyancy driven planetary-boundary-layer flows. J. Atmos. Sci., 51, 9991022.

Noh, Y., H. S. Min, and S. Raasch, 2004: Large eddy simulation of the ocean mixed layer: The effects of wave breaking and Langmuir circulation. J. Phys. Oceanogr., 34, 720-735.
Polton, J. A., D. M. Lewis, and S. E. Belcher, 2005: The role of wave-induced Coriolis-Stokes forcing on the wind-driven mixed layer. J. Phys. Oceanogr., 35, 444-457.

Powell, M. D., P. J. Vickery, and T. A. Reinhold, 2003: Reduced drag coefficient for high wind speeds in tropical cyclones. Nature, 422, 279-283.

Price, J. F., 1981: Upper-ocean response to a hurricane. J. Phys. Oceanogr., 11, 153-175.

— R. A. Weller, and R. Pinkel, 1986: Diurnal cycling: Observations and models of the upper ocean response to diurnal heating, cooling, and wind mixing. J. Geophys. Res., 91, 8411-8427.

Romero, L., and W. K. Melville, 2010a: Airborne observations of fetch-limited waves in the Gulf of Tehuantepec. J. Phys. Oceanogr., 40, 441-465.

- and - 2010b: Numerical modeling of fetch-limited waves in the Gulf of Tehuantepec. J. Phys. Oceanogr., 40, 466-486.

Sanford, T. B., J. F. Price, J. B. Girton, and D. C. Webb, 2007: Highly resolved observations and simulations of the ocean response to a hurricane. Geophys. Res. Lett., 34, L13604, doi:10.1029/2007GL029679.

$\longrightarrow,-$, and - , 2011: Upper-ocean response to Hurricane Frances (2004) observed by profiling EM-Apex floats. J. Phys. Oceanogr., 41, 1041-1056.

Schmidt, H., and U. Schumann, 1989: Coherent structure of the convective boundary layer. J. Fluid Mech., 200, 511-562.

Skyllingstad, E. D., and D. W. Denbo, 1995: An ocean large-eddy simulation of Langmuir circulations and convection in the surface mixed layer. J. Geophys. Res., 100, 8501-8522.

— - W. D. Smyth, J. N. Moum, and H. Wijesekera, 1999: Upperocean turbulence during a westerly wind burst: A comparison of large-eddy simulation results and microstructure measurements. J. Phys. Oceanogr., 29, 5-28.

, - - and G. B. Crawford, 2000: Resonant wind-driven mixing in the ocean boundary layer. J. Phys. Oceanogr., 30, 219-237.

Smith, J. A., 1998: Evolution of Langmuir circulation during a storm. J. Geophys. Res., 103 (C6), 12 649-12 668.

Snyder, R. L., F. W. Dobson, J. A. Elliott, and R. B. Long, 1981: Array measurements of atmospheric pressure fluctuations above surface gravity waves. J. Fluid Mech., 102, 1-59.

Sullivan, P. P., and J. C. McWilliams, 2010: Dynamics of winds and currents coupled to surface waves. Annu. Rev. Fluid Mech., 42, $19-42$.

, and E. G. Patton, 2011: The effect of mesh resolution on convective boundary-layer statistics and structures generated by large-eddy simulation. J. Atmos. Sci., 68, 2395-2415.

- J. C. McWilliams, and C.-H. Moeng, 1994: A subgrid-scale model for large-eddy simulation of planetary boundary-layer flows. Bound.-Layer Meteor., 71, 247-276.

- C.-H. Moeng, B. Stevens, D. H. Lenschow, and S. D. Mayor, 1998: Structure of the entrainment zone capping the convective atmospheric boundary layer. J. Atmos. Sci., 55, 3042-3064. J. C. McWilliams, and W. K. Melville, 2004: The oceanic boundary layer driven by wave breaking with stochastic variability. I: Direct numerical simulations. J. Fluid Mech., 507, 143-174.

- _ - and —_, 2007: Surface gravity wave effects in the oceanic boundary layer: Large-eddy simulation with vortex force and stochastic breakers. J. Fluid Mech., 593, 405452.

Teixeira, M. A. C., and S. E. Belcher, 2002: On the distortion of turbulence by a progressive surface wave. J. Fluid Mech., 458, 229-267. 
Terray, E. A., M. A. Donelan, Y. C. Agrawal, W. M. Drennan, K. K. Kahma, A. J. Williams III, Hwang P. A., and S. A. Kitaigorodskii, 1996: Estimates of kinetic energy dissipation under breaking waves. J. Phys. Oceanogr., 26, 792-807.

Tolman, H. L., 2002: User manual and system documentation of Wavewatch-III, version 2.22. NOAA/NWS/NCEP/MMAB Tech. Rep. Tech. Note 222, 133 pp.

Van Roekle, L. P., B. Fox-Kemper, P. P. Sullivan, P. E. Hamlington, and S. R. Haney, 2012: The form and orientation of Langmuir cells for misaligned winds and waves. J. Geophys. Res., 117, C05001, doi:10.1029/2011JC007516.

van Vledder, G. P., 2006: The WRT method for the computation of non-linear four-wave interactions in discrete spectral wave models. Coastal Eng., 53, 223-242.

Wright, C., and Coauthors, 2001: Hurricane directional wave spectrum spatial variation in the open ocean. J. Phys. Oceanogr., 31, 2472-2488.
Young, I. R., 1998: Observations of the spectra of hurricane generated waves. Ocean Eng., 25 (4-5), 261-276.

- 2003: A review of the sea state generated by hurricanes. Mar. Struct., 16, 201-218.

_ 2006: Directional spectra of hurricane wind-waves. J. Geophys. Res., 111, C08020, doi:10.1029/2006JC003540.

Zedler, S. E., 2007: Strong wind forcing of the ocean. Ph.D. thesis, University of California, San Diego, $127 \mathrm{pp}$.

—_, T. D. Dickey, S. C. Doney, J. F. Price, X. Xu, and G. L. Mellor, 2002: Analyses and simulations of the upper ocean's response to Hurricane Felix at the Bermuda Testbed Mooring site: 13-23 August 1995. J. Geophys. Res., 107, $1-29$.

_ - P. P. Niiler, D. Stammer, E. Terrill, and J. Morzel, 2009: Ocean's response to Hurricane Frances and its implications for drag coefficient parameterizations at high wind speeds. J. Geophys. Res., 114, C04016, doi:10.1029/2008JC005205. 